\title{
Forecasting Return of Used Products for Remanufacturing Using Graphical Evaluation and Review Technique (GERT)
}

Li Zhou ${ }^{1}$, Jiaping Xie ${ }^{2 *}$, Xiaoyu Gu ${ }^{1}$, Yong Lin $^{1}$, Petros Ieromonachou ${ }^{1}$, Xiaole Zhang ${ }^{1}$

1. Faculty of Business, University of Greenwich, London, SE10 9LS, UK

\section{Li.Zhou@gre.ac.uk}

2*. College of Business, Shanghai University of Finance and Economics, Shanghai, P.R. China

Jiaping@mail.shufe.edu.cn; jiaping2004@ hotmail.com 
Highlights (for review)

Highlights:

1. A forecasting model by using GERT stochastic network analysis technique;

2. The model is generalised to be applicable to any product structure;

3. The model can predict product return quantity, probability, and expected time;

4. The model can also predict parts, components, materials and disposal in the same manner. 


\title{
Forecasting Return of Used Products for Remanufacturing Using Graphical Evaluation and Review Technique
}

\begin{abstract}
This research develops a forecasting model that can predict the quantity, time and probability of product return, recyclable parts/components/materials and disposal. It adopts the Graphical Evaluation and Review Technique (GERT) by translating the remanufacturing operational process into a stochastic network. This stochastic network possesses two characteristics: activities having a probability of occurrence associated with them; and time to perform an activity. Together with the GERT method, Mason's rule is applied to calculate the equivalence transfer function of the system, therefore predicting the desired outcomes. A generic eight-step process on how to implement this method in any structure of return products and remanufacturing network is provided. A numerical example is presented to demonstrate the result of using GERT on forecasting printer remanufacturing outcomes. The main contribution of this research is: Instead of giving one result such as either return quantity, or time, or probability, our research can forecast three of these outcomes simultaneously, and the algorithm is generalised to be applicable to any product structure and remanufacturing network.
\end{abstract}

Keywords: return forecasting, GERT, product return, remanufacturing, Moment-Generating Function, transfer function. 


\section{Introduction}

The challenges of sustainable production concern primarily the energy consumption and the subsequent challenges associated with reducing the carbon footprint, other forms of pollution, natural resource depletion, waste management and landfill space. In this specific area, the focus on sustainability has created a plethora of terms often used to represent the same concept such as eco-efficiency, remanufacturing, green technology, cleaner production etc. Glavic and Lukman (2007) explain the semantic differences between the various terms. Achieving eco-efficient production and consumption systems requires 'closing the loop' to create circular systems. To do so, Lund (1984) proposed the concept of remanufacturing. According to Lund (1984), remanufacturing is interpreted as a full production process that transfers a worn, durable product into both a useful and an economic state. Remanufacturing as a key enabler to sustainable production plays a strategic role in waste management and environmentally conscious production (Ijomah et al., 2007). From manufacturers' point of view, the economic efficiency of remanufacturing is clear and such systems would also be more eco-efficient. Kerra and Ryan (2001) studied Xerox photocopiers in Australia and found that remanufacturing can reduce resource consumption and waste generation over the life cycle of a photocopier.

However, managing remanufacturing operations has proved to be challenging (Zhou et al., 2006). Many scholars have studied the inherent complexity and uncertainty of the remanufacturing system. Guide and Srivastava (1997) suggested that the remanufacturing system is a complex system. To cope with uncertainty there is a need 
for accurate and up-to-date information, including the remanufacturing rate of materials and the frequency of using a specific processing route. Guide (2000) investigated production practices in American remanufacturing enterprises to identify multiple uncertainties and their impact on remanufacturing production, such as uncertainty of return of product, dismantling of parts, remanufacturing rate of materials, lead time of remanufacturing, and imbalance between recycling and demand. These uncertainties bring a big challenge in managing a remanufacturing system. Therefore, to plan remanufacturing production effectively, forecasting of product return and their time must be in place.

Conventionally, forecasting models can be classified into qualitative and quantitative approaches. The former is based on subjective judgement when historical data are unavailable. The latter is dominated by practice. Some commonly used quantitative forecasting methods include time series methods, e.g. moving average, linear prediction; causal/econometric forecasting methods, e.g. regression analysis and autoregressive moving average with exogenous input; judgemental methods, e.g. Delphi method, statistical survey; artificial intelligence methods, e.g. artificial neural networks, data mining, machine learning; and others such as simulation (Wikepedia, 2015). Nevertheless, since there are many uncertainties in a remanufacturing system, it is difficult to make conventional forecasting methods play a role, in view of the current situation in which research on product return predictions is still scarce (Andrew-munot et al., 2015; Fleischmann et al., 1997; Kelle and Silver, 1989). The importance of forecasting is significant in remanufacturing operations. To 
construct a profitable remanufacturing process and reverse logistics system, its capacity planning, remanufacturing scheduling, inventory management, network design and resourcing allocation heavily rely on the amount of product return. These strategic plans are primarily based on return forecasting. In a recently published literature review by Govindan et al. (2014), return forecasting has been identified one of research gaps that needs to be addressed. This motivates us to develop a new approach that can be used in forecasting returns for remanufacturing production. The proposed model is able to predict products' return quantity, possibility and time through applying the theories of stochastic networks and feedback control systems (Pritsker and Happ, 1966; Samuel, 1956). Furthermore, it also can estimate the amount and time of detachable parts based on product structure. The result of this paper contributes to the literature of return forecasting methods and we hope it could help responsible manufacturers and remanufacturers to improve the efficiency of production scheduling through forecasting returns.

Section 2 reviews the relevant literature on returns and forecasting methods. The proposed Graphical Evaluation and Review Technique (GERT) methodology with Mason's rules is described in Section 3. By using this methodology, Section 4 provides more details on how to apply GERT on analysing a product return and remanufacturing stochastic network, followed by a numerical example in Section 5 . Section 6 concludes.

\section{Literature review}

2.1 Probability distribution of product returns 
Despite uncertainties, distribution of product return is expected to obey a certain type of random distribution. Thierry (1993) stated that the uncertainties of return time, quantity and quality have a considerable impact on remanufacturing production planning and optimisation. García-Alvarado et al. (2015) and Decroix et al. (2005) assumed that product returns conform to a discrete distribution. Some researchers consider product return or processing time to obey a Poisson distribution (Bayındir et al., 2003; Zhao et al., 2015; Kiesmüller and van der Laan, 2001; Toktay et al., 2000). De Brito and Dekker (2004) suggested that if market demand is subject to a homogeneous Poisson distribution, the return rate of discarded products will also conform to the same distribution.

The majority of returned products are due to malfunction or breakdown. Most of the existing research treats machine breakdown time as a negative exponential distribution (Christer and Waller, 2015; Fu et al., 2015; Ke and Wang, 1999; Taylor and Andrushchenko, 2014). It refers to the life cycle of a non-aging product, i.e. one not totally worn but one that fails to function for some reason, before it reaches to the expected end of life. This indirectly implies that the time of multiple uses of product should also obey a negative exponential distribution, as adopted in this research.

\subsection{Forecasting models on return quantity}

It is quite common that product return is assumed to be independent of demand (García-Alvarado et al., 2015; Richter, 1996a, 1996b), while others believe that there are some links between return and demand (Kelle and Silver, 1989; Kiesmüller and van der Laan, 2001; Toktay et al., 2000). 
Kiesmüller and van der Laan (2001) and Kelle and Silver (1989) considered the situation where return product quantity is probability correlated with product demand. Toktay et al. (2000) predicted probability densities for the return probability and the return delay based on real-time sales and return data by applying Bayesian statistics and survival analysis. De Brito and van der Laan (2009) suggested that it is reasonable to select historical information about sales and returns in terms of products' characteristics when predicting return quantity.

Carrasco-Gallego and Ponce-Cueto (2009) suggest that univariate time series models would be useful when only data available are historic return series in a linear reverse logistics system. In contrast, when available data are in a wider range variety, dynamic regression models would be more suitable (Kumar and Yamaoka, 2007). This type of models can capture the relationship among different activities and illustrate the consequence of various adopted strategies. Hanafi et al. (2008) adopt fuzzy Coloured Petri Net (CPN) approach to simulate product return network and forecast time and location of returns. It is noted that $\mathrm{CPN}$ was initially developed for project management, which then extended to various comprehensive techniques, one of them is GERT.

Similar to Hanafi et al. (2008) and Kumar and Yamaoka (2007), in this paper we adopt GERT approach to capture the dynamic process of remanufacturing operations to predict return quantity, time and probability.

2.3 Prediction of parts disassembly 
This refers to predicting the time and availability of disassembling parts. The fluctuation of disassembly time is usually reflected in operations time variance, which is not simply accumulated by adding up operations time among different processes. Therefore, the uncertainty of remanufacturing has deepened. Lye et al. (2000) used the shortest path algorithm to optimise the disassembly and reassembly sequence. Gungor and Gupta (2001) applied a branch-and-bound algorithm to predict the disassembly of products. However, calculation of these methods is extremely time consuming. In this paper, the adopted GERT together with Mason's rule simplifies the calculation by using Laplace transfer functions.

\subsection{Graphical Evaluation and Review Technique (GERT) and its application}

GERT was first developed by Pritsker and Happ (1966). 'It is a procedure for ... (1) analysing networks that contained activities that had a probability of occurrence associated with them, and (2) treating the plausibility that the time to perform an activity was not a constant, but a random variable ... Networks containing these two elements were described by the term 'stochastic networks"' (Pritsker, 1966, p. iii). This technique was initially developed for managing the Apollo project, which was then widely adopted in project management such as risk management (Ahmed et al., 2007), dynamic scheduling (Pena-Mora and Li, 2001), managing uncertainty (De Meyer et al., 2002) and many others.

In the last three decades, the applications of GERT have been extended to other areas. For instance, Fisher and Goldstein (1983) applied a similar technique in analysing cognition behaviour; and Kosugi et al. (2004) applied GERT to evaluate energy 
efficiency in the R\&D projects of $\mathrm{CO}_{2}$ capture technologies. Five technologies were studied and their R\&D processes were analogised into stochastic networks with the above-mentioned two characteristics; that is, probability and a random variable. By using GERT, they predicted the time of project completion, success probabilities and cost effectiveness of investment.

In system engineering, stochastic network modelling is a technique for analysing the events of a process with branches of probability and closed loops. It is effective for prediction and planning when uncertainty exists among different operations. Through analysis of the transition function between nodes of stochastic networks, recovery network models with multi-starts to multi-terminals and multi-loops can be analysed. Xie et al. (2007) built a forecasting model for reclaiming and reusing products based on GERT network theory. Through the GERT method, the average time of every disassembly process and the availability rate of parts were derived. However, the model was designed for a specific product structure, which would be hard to apply in general. Zhou et al. (2010) justified that product return and remanufacturing (PRR) networks have the same characteristics as stochastic networks: multiple loops because of product use and reuse; branches and loops for different remanufacturing activities; uncertainty and randomness in the process; the entire network having more than one start point and end point. Hence, applying GERT in the analysis of these PRR networks is feasible and viable. They developed a product return forecasting model by using the GERT technique, and figured out the quantity and timing of remanufacturable parts and components as well as recyclable materials. However, the 
model did not take into account product return and resale in first- and second-hand markets, and in particular the decision on remanufacturing activities was limited, without including disposal.

This paper develops a generic forecasting model of product return and remanufacturing quantity, time and probability. It can be applied to any type of products provided that the $\mathrm{BOM}$ is known. We start by mapping the product return and remanufacturing process, as shown in Figure 1. This is then translated into a GERT network, as shown in Figure 2. Based on the structure of the network, a series of process transfer functions are derived to calculate quantity, time and probability.

\section{Description of GERT methodology: A generic eight-step process}

The inputs of the GERT are the parameters for each activity. These parameters include: the probability from activity (node) i to activity (node) $\mathrm{j}$, estimated time period for each activity and the product original structure. In this case, the outputs are the prediction of product return quantity, timing and probability, salvageable parts/components/materials' quantity, timing and probability, and finally disposal.

To generalise and also simplify the process, the methodology can be described in eight steps:

Step 1: Mapping the process in order to derive the causal flow chart;

Step 2: Translating the flow chart into the stochastic network;

Step 3: Estimating each activity's parameters (from node $i$ to node $j$ ): probability $P_{i j}$ and time probability density function $\mathrm{f}\left(\mathrm{t}_{\mathrm{i}}\right)$; 
Step 4: Integrating the two parameters of each activity $(\mathrm{i}, \mathrm{j})$ into one transfer function $w_{i j}(s): w_{i j}(s)=P_{i j} M_{i j}(s)$

Where $\mathrm{M}_{\mathrm{ij}}(\mathrm{s})=\mathrm{E}\left(\mathrm{e}^{\mathrm{t}_{\mathrm{i}}^{\mathrm{s}}}\right)=\int_{-\infty}^{+\infty} \mathrm{e}^{\mathrm{t}_{\mathrm{i}} \mathrm{s}} \mathrm{f}\left(\mathrm{t}_{\mathrm{i}}\right) \mathrm{dt}_{\mathrm{i}}$ is a moment-generating function (MGF).

Step 5: Applying Mason's rules (Mason, 1956a) - details explained in 4.4.2 calculate total equivalence transfer function $\mathrm{W}_{\mathrm{E}_{0 \mathrm{n}}}$ from initial node 0 to node $\mathrm{j}$ based on the network structure and the value of $\mathrm{w}_{\mathrm{ij}}(\mathrm{s})$. This is a rather complex calculation but indeed a key stage;

Step 6: According to the definition of MGF, the probability $\left(\mathrm{P}_{\mathrm{E}_{0 j}}\right)$ of the activity from initial node 0 to $\mathrm{j}$ is $\mathrm{P}_{\mathrm{E}_{0 j}}=\left.\mathrm{W}_{\mathrm{E}_{0 j}}(\mathrm{~s})\right|_{\mathrm{s}=0}$;

Step 7: According to the characteristics of MGF (Pishro-Nik, 2016), the expected return time from initial node 0 to node $j$ is: $\mathrm{E}(\mathrm{t})=\mu_{1}=\left.\frac{\partial}{\partial \mathrm{s}}\left[\mathrm{M}_{\mathrm{E}_{0 \mathrm{n}}}(\mathrm{s})\right]\right|_{\mathrm{s}=0}=\left.\frac{1}{\mathrm{P}_{\mathrm{E}_{0 \mathrm{n}}}} \frac{\partial}{\partial \mathrm{s}}\left[\mathrm{W}_{\mathrm{E}_{0 \mathrm{n}}}(\mathrm{s})\right]\right|_{\mathrm{s}=0} ;$

Step 8: The predicted quantity of product return/parts/components/materials equals to the probability of each activity multiple total amount of sold product: $\mathrm{P}_{\mathrm{E}_{0 j}} \times$ Sales . In the following section, further details on how to calculate each activity and the desired outcomes are provided.

\section{Analysis of the GERT remanufacturing networks}

\subsection{Mapping the process of product return and remanufacturing}

As shown in Figure 1, when a consumer receives a product, if he/she is not satisfied with the product it can be returned to the retailer. The returned products will be tested 
and classified into directly resellable or reconditionable and for sale back to the first-hand market. This is a common practice in mobile phones and laptops, for instance refurbished Apple Macs; there might be some products going to the second-hand market at a lower price. In this research, to simplify the case, we assume that retailers are involved in both first- and second-hand markets. If a product is not resellable, it will be sent to a remanufacturer to undertake sorting and testing. Based on return quality, remanufacturing processes include: (a) disassembling product into parts, reconditioning and resale; (b) disassembling parts into components, reconditioning and resale; (c) extracting useful materials from components and sale to a material market; and (d) disposal.

Insert Figure 1 here.

\subsection{GERT Stochastic Network}

In the GERT network, each node is presented by a different icon. In inputs, there are three types of relationship: XOR, OR and AND. Outputs have two types of relationships: non-deterministic and deterministic. By combining input and output, there are six logic nodes, as shown in Table 1.

Insert Table 1 here.

In the GERT network, a line with an arrow means a job and each line has two parameters: probability, which means the possibility of doing the job; and time, which means the duration of the job.

The network for product return and remanufacturing is shown in Figure 2. For each 
node $i$, the process can be classified as three types, the distribution times of which are:

- Activity 1: sales from new product launch to exit market. We assume that the process time obeys a normal distribution $\mathrm{N}\left(\mu, \sigma^{2}\right)$, which reflects the curve of the product life cycle.

- Activity 2: multiple uses of products. We assume that the multiple usage time $\mu_{i}$ is a negative exponential distribution given the fact that it is in correlation with product breakdowns (Christer and Waller, 2015; Fu et al., 2015; Ke and Wang, 1999; Taylor and Andrushchenko, 2014).

- Activity 3: remanufacturing-related activities include sorting, testing, dismantling and so on. Without loss of generosity, to simplify the mathematical process, we assume that the process time $t_{i}$ is a constant (Xie et al., 2007).

The parameter $P_{i j}$ refers to the probability of state $i$ transitioning to state $j$ with the value of a $\mathrm{P}$. The superscripts $\mathrm{p}, \mathrm{c}$ and $\mathrm{m}$ mean that the product can be dismantled into a number of $p$ parts, $c$ components and $m$ types of materials, respectively.

Insert Figure 2 here.

\subsection{Notation}

The description of each node is shown Table 2:

Insert Table 2 here.

The transition process is shown in Table 3 . 


\subsection{Mathematical model}

\subsubsection{Moment-Generating Function (MGF)}

The Moment-Generating Function (MGF) is used to describe a random variable's probability distribution as an alternative specification. Compared with the cumulative distribution function (CDF) or probability density function (PDF), MGF offers a different method of analysing results through the weighted sum of random variables (Wikipedia). The MGF of a random variable $t$ is defined:

$$
\mathrm{M}_{\mathrm{t}}(\mathrm{s})=\mathrm{E}\left(\mathrm{e}^{\mathrm{ts}}\right), \quad \mathrm{s} \in \mathrm{R}
$$

wherever this expectation exists.

In the PRR network, assuming that the density function is continuous and the completion time's density function at node $i$ is $f\left(t_{i}\right)$, then the MGF of $t_{i}$ is the MGF of the arrow from $\mathrm{i}$ to $\mathrm{j}$, which is

$$
M_{i j}(s)=E\left(e^{t_{i} s}\right)=\int_{-\infty}^{+\infty} e^{t_{i} s} f\left(t_{i}\right) d t_{i}
$$

If $t_{i}$ is constant, the MGF is

$$
\mathrm{M}_{\mathrm{ij}}(\mathrm{s})=\mathrm{e}^{\mathrm{t}_{\mathrm{i}} \mathrm{s}}
$$

If $t_{i}$ obeys a negative exponential distribution, the MGF is

$$
\mathrm{M}_{\mathrm{ij}}(\mathrm{s})=\frac{1}{1-\mu_{\mathrm{i}} \mathrm{s}}
$$

While if $\mathrm{t}_{\mathrm{i}}$ obeys the normal distribution $\mathrm{N}\left(\mu, \sigma^{2}\right)$, the MGF is

$$
\mathrm{M}_{\mathrm{ij}}(\mathrm{s})=\mathrm{e}^{\mu_{\mathrm{i}} \mathrm{s}+\frac{1}{2} \sigma^{2} \mathrm{~s}^{2}}
$$




\subsubsection{Mason equivalence principle}

The equivalence transfer function from any node $\mathrm{i}$ to node $\mathrm{j}$ can be obtained by using Mason's rules (Mason, 1956b):

$$
\mathrm{W}_{\mathrm{E}_{\mathrm{ij}}}(\mathrm{s})=\frac{\sum_{\mathrm{l}=1}^{\mathrm{n}} \mathrm{G}_{1} \Delta_{1}}{\Delta}
$$

where $G_{1}$ is the gain of the $1^{\text {th }}$ forward route from i to $\mathrm{j}$, and $\Delta_{1}$ is the loop gain of the $1^{\text {th }}$ loop, $1 \in\{1, n\}$, and must be an integer. In control theory, 'gain' means the path or loop's transfer function i.e. output is divided by input.

$\Delta$ is the determinant of the graph, where

$$
\Delta=1-\sum \mathrm{L}_{\mathrm{x}}+\sum \mathrm{L}_{\mathrm{x}} \mathrm{L}_{\mathrm{y}}-\sum \mathrm{L}_{\mathrm{x}} \mathrm{L}_{\mathrm{y}} \mathrm{L}_{\mathrm{z}}+\ldots+(-1)^{\mathrm{k}} \sum \ldots+\ldots
$$

Where L refers to a loop

$\sum \mathrm{L}_{\mathrm{x}}$ is the sum of the transfer coefficient for different loops

$\sum \mathrm{L}_{\mathrm{x}} \mathrm{L}_{\mathrm{y}}$ is the sum of the transfer coefficient for two non-touch loops

$\sum \mathrm{L}_{\mathrm{x}} \mathrm{L}_{\mathrm{y}} \mathrm{L}_{\mathrm{z}}$ is the sum of the transfer coefficient for three non-touch loops

$(-1)^{\mathrm{k}} \sum \ldots$ is the sum of the transfer coefficient for $\mathrm{k}$ non-touch loops

4.4.3 Transfer functions for the product recycling process

The process must account from initial node 0. Through using (3), (4) and (5), the MGF for each state transition is

$$
\mathrm{M}_{01}(\mathrm{~s})=\mathrm{e}^{\mathrm{t}_{0} \mathrm{~s}} \quad, \quad \mathrm{M}_{12}(\mathrm{~s})=\mathrm{M}_{13}(\mathrm{~s})=\mathrm{e}^{\mu_{0} \mathrm{~s}+\frac{1}{2} \sigma^{2} \mathrm{~s}^{2}} \quad, \quad \mathrm{M}_{21}(\mathrm{~s})=\mathrm{e}^{\mathrm{t}_{1} \mathrm{~s}} \quad,
$$




$$
\begin{aligned}
& M_{33}(s)=M_{34}(s)=\frac{1}{1-\mu_{1} s} \quad, \quad M_{45}(s)=M_{47}(s)=e^{t_{2} s} \quad, \quad M_{56}(s)=e^{t_{3} s} \\
& M_{66}(s)=M_{67}(s)=\frac{1}{1-\mu_{2} s} \quad, \quad M_{78}(s)=M_{7,11}(s)=M_{7,15}(s)=e^{t_{4} s} \\
& M_{89}(s)=M_{8,10}(s)=M_{8,11}(s)=e^{t_{5} s} \\
& M_{10,11}^{c}(s)=M_{10,13}^{c}(s)=e^{t_{7}^{c} s}, \quad M_{11,14}^{\mathrm{m}}(s)=M_{11,15}^{m}(s)=e^{t_{8}^{m_{8}} s}
\end{aligned}
$$

The transfer function for arrow $(i, j)$ is described below:

$$
\mathrm{W}_{\mathrm{ij}}(\mathrm{s})=\mathrm{P}_{\mathrm{ij}} \mathrm{M}_{\mathrm{ij}}(\mathrm{s})
$$

Therefore, the transfer function for each state transition is

$$
\begin{aligned}
& \mathrm{w}_{01}(\mathrm{~s})=\mathrm{e}^{\mathrm{t}_{0} \mathrm{~s}}, \quad \mathrm{w}_{12}(\mathrm{~s})=\left(1-\mathrm{P}_{1}\right) \mathrm{e}^{\mu_{0} \mathrm{~s}+\frac{1}{2} \sigma^{2} \mathrm{~s}^{2}}, \quad \mathrm{w}_{13}(\mathrm{~s})=\mathrm{P}_{1} \mathrm{e}^{\mu_{0} \mathrm{~s}+\frac{1}{2} \sigma^{2} \mathrm{~s}^{2}}, \quad \mathrm{w}_{21}(\mathrm{~s})=\mathrm{e}^{\mathrm{t}_{1} \mathrm{~s}}, \\
& \mathrm{w}_{33}(\mathrm{~s})=\frac{\mathrm{P}_{2}}{1-\mu_{1} \mathrm{~s}}, \quad \mathrm{w}_{34}(\mathrm{~s})=\frac{\mathrm{P}_{3}}{1-\mu_{1} \mathrm{~s}}, \quad \mathrm{w}_{45}(\mathrm{~s})=\mathrm{P}_{4} \mathrm{e}^{\mathrm{t}_{2} \mathrm{~s}}, \quad \mathrm{w}_{47}(\mathrm{~s})=\left(1-\mathrm{P}_{4}\right) \mathrm{e}^{\mathrm{t}_{2} \mathrm{~s}}, \quad \mathrm{w}_{56}(\mathrm{~s})=\mathrm{e}^{\mathrm{t}_{3} \mathrm{~s}}, \\
& \mathrm{w}_{66}(\mathrm{~s})=\frac{\mathrm{P}_{5}}{1-\mu_{2} \mathrm{~s}} \quad, \quad \mathrm{w}_{67}(\mathrm{~s})=\frac{\mathrm{P}_{6}}{1-\mu_{2} \mathrm{~s}}, \quad \mathrm{w}_{78}(\mathrm{~s})=\mathrm{P}_{7} \mathrm{e}^{\mathrm{t}_{4} \mathrm{~s}}, \quad \mathrm{w}_{7,11}(\mathrm{~s})=\mathrm{P}_{8} \mathrm{e}^{\mathrm{t}_{4} \mathrm{~s}}, \\
& \mathrm{w}_{7,15}(\mathrm{~s})=\left(1-\mathrm{P}_{7}-\mathrm{P}_{8}\right) \mathrm{e}^{\mathrm{t}_{4} \mathrm{~s}}, \mathrm{w}_{89}(\mathrm{~s})=\mathrm{P}_{9} \mathrm{e}^{\mathrm{t}_{5} \mathrm{~s}}, \quad \mathrm{w}_{8,10}(\mathrm{~s})=\mathrm{P}_{10} \mathrm{e}^{\mathrm{t}_{5} \mathrm{~s}}, \quad \mathrm{w}_{8,11}(\mathrm{~s})=\left(1-\mathrm{P}_{9}-\mathrm{P}_{10}\right) \mathrm{e}^{\mathrm{t}_{5} \mathrm{~s}}, \\
& \mathrm{w}_{9,10}^{\mathrm{p}}(\mathrm{s})=\left(1-\mathrm{P}_{11}^{\mathrm{p}}\right) \mathrm{e}^{\mathrm{t}_{6}^{\mathrm{p}} \mathrm{s}}, \quad \mathrm{w}_{9,12}^{\mathrm{p}}(\mathrm{s})=\mathrm{P}_{11}^{\mathrm{p}} \mathrm{e}^{\mathrm{t}_{6}^{\mathrm{p} s}}, \quad \mathrm{w}_{10,11}^{\mathrm{c}}(\mathrm{s})=\left(1-\mathrm{P}_{12}^{\mathrm{c}}\right) \mathrm{e}^{\mathrm{t}_{7}^{\mathrm{c}} \mathrm{s}}, \quad \mathrm{w}_{10,13}^{\mathrm{c}}(\mathrm{s})=\mathrm{P}_{12}^{\mathrm{c}} \mathrm{e}^{\mathrm{t}_{7}^{\mathrm{c} s}}, \\
& \mathrm{w}_{11,14}^{\mathrm{m}}(\mathrm{s})=\mathrm{P}_{13}^{\mathrm{m}} \mathrm{e}^{\mathrm{t}_{8}^{\mathrm{m} s}}, \quad \mathrm{w}_{11,15}^{\mathrm{m}}(\mathrm{s})=\left(1-\mathrm{P}_{13}^{\mathrm{m}}\right) \mathrm{e}^{\mathrm{t}_{8}^{\mathrm{m}} \mathrm{s}} .
\end{aligned}
$$

\subsubsection{Forecasting product return}

From state 0 to state 7 , there are 2 paths, which are $0 \rightarrow 1 \rightarrow 3 \rightarrow 4 \rightarrow 7$ and $0 \rightarrow 1 \rightarrow 3 \rightarrow 4$

$\rightarrow 5 \rightarrow 6 \rightarrow 7$. Its characteristic formula is

$$
\begin{aligned}
\Delta & =1-\mathrm{w}_{12} \mathrm{w}_{21}-\mathrm{w}_{33}-\mathrm{w}_{66}+\mathrm{w}_{12} \mathrm{w}_{21} \mathrm{w}_{33}+\mathrm{w}_{12} \mathrm{w}_{21} \mathrm{w}_{66}+\mathrm{w}_{33} \mathrm{w}_{66}-\mathrm{w}_{12} \mathrm{w}_{21} \mathrm{w}_{33} \mathrm{w}_{66} \\
& =\left(1-\mathrm{w}_{12} \mathrm{w}_{21}\right)\left(1-\mathrm{w}_{33}\right)\left(1-\mathrm{w}_{66}\right)
\end{aligned}
$$

and by using (6) for simplification, the equivalence transfer function is 


$$
\begin{aligned}
\mathrm{W}_{\mathrm{E}_{07}}(\mathrm{~s}) & =\frac{\mathrm{w}_{01}\left(\mathrm{w}_{13} \mathrm{~W}_{34} \mathrm{~W}_{47}\left(1-\mathrm{w}_{66}\right)+\mathrm{w}_{13} \mathrm{~W}_{34} \mathrm{w}_{45} \mathrm{~W}_{56} \mathrm{~W}_{67}\right)}{\left(1-\mathrm{w}_{12} \mathrm{~W}_{21}\right)\left(1-\mathrm{w}_{33}\right)\left(1-\mathrm{w}_{66}\right)} \\
& =\frac{\mathrm{w}_{01}}{\left(1-\mathrm{w}_{12} \mathrm{~W}_{21}\right)} \cdot \frac{\mathrm{w}_{13} \mathrm{~W}_{34}}{\left(1-\mathrm{w}_{33}\right)} \cdot\left(\mathrm{w}_{47}+\frac{\mathrm{w}_{45} \mathrm{~W}_{56} \mathrm{~W}_{67}}{\left(1-\mathrm{w}_{66}\right)}\right) \\
& =\frac{\mathrm{e}^{\mathrm{t}_{0} \mathrm{~s}}}{\left(1-\left(1-\mathrm{P}_{1}\right) \mathrm{e}^{\mathrm{t}_{1} \mathrm{~s}+\mu_{0} \mathrm{~s}+\sigma^{2} \mathrm{~s}^{2} / 2}\right)} \cdot \frac{\mathrm{P}_{1} \mathrm{P}_{3} \mathrm{e}^{\mu_{0} \mathrm{~s}+\sigma^{2} \mathrm{~s}^{2} / 2}}{\left(1-\mu_{1} \mathrm{~s}-\mathrm{P}_{2}\right)} \cdot\left(\left(1-\mathrm{P}_{4}\right) \mathrm{e}^{\mathrm{t}_{2} \mathrm{~s}}+\frac{\mathrm{P}_{4} \mathrm{P}_{6} \mathrm{e}^{\left(\mathrm{t}_{2}+\mathrm{t}_{3}\right) \mathrm{s}}}{\left(1-\mu_{2} \mathrm{~s}-\mathrm{P}_{5}\right)}\right) \\
& =\frac{\mathrm{P}_{1} \mathrm{P}_{3} \mathrm{e}^{\mathrm{t}_{0} \mathrm{~s}+\mu_{0} \mathrm{~s}+\sigma^{2} \mathrm{~s}^{2} / 2}}{\left(1-\left(1-\mathrm{P}_{1}\right) \mathrm{e}^{\mathrm{t}_{1} \mathrm{~s}+\mu_{0} \mathrm{~s}+\sigma^{2} \mathrm{~s}^{2} / 2}\right)\left(1-\mu_{1} \mathrm{~s}-\mathrm{P}_{2}\right)} \cdot\left(\left(1-\mathrm{P}_{4}\right) \mathrm{e}^{\mathrm{t}_{2} \mathrm{~s}}+\frac{\mathrm{P}_{4} \mathrm{P}_{6} \mathrm{e}^{\left(\mathrm{t}_{2}+\mathrm{t}_{3}\right) \mathrm{s}}}{\left(1-\mu_{2} \mathrm{~s}-\mathrm{P}_{5}\right)}\right)
\end{aligned}
$$

Let $\mathrm{s}=0, \quad \mathrm{~W}_{\mathrm{E}_{0 j}}(0)=\mathrm{P}_{\mathrm{E}_{0 j}} \mathrm{M}_{\mathrm{Ej}}(0)=\left.\mathrm{P}_{\mathrm{E}_{0 \mathrm{j}}} \int_{-\infty}^{+\infty} \mathrm{e}^{\mathrm{ts}} \mathrm{f}(\mathrm{t}) \mathrm{dt}\right|_{\mathrm{s}=0}=\mathrm{P}_{\mathrm{E}_{0 j}}$ and the expected time from 0 to $\mathrm{j}$ is the first-order derivation of MGF.

Therefore, the probability of product return is:

$$
\mathrm{P}_{\mathrm{E}_{07}}=\frac{\mathrm{P}_{3}}{\left(1-\mathrm{P}_{2}\right)} \cdot\left(\left(1-\mathrm{P}_{4}\right)+\frac{\mathrm{P}_{4} \mathrm{P}_{6}}{\left(1-\mathrm{P}_{5}\right)}\right)
$$

With the expected time:

$$
\mathrm{t}_{\mathrm{E}_{0 \mathrm{n}}}=\mathrm{E}(\mathrm{t})=\left.\frac{\partial}{\partial \mathrm{s}}\left[\mathrm{M}_{\mathrm{E}_{0 \mathrm{n}}}(\mathrm{s})\right]\right|_{\mathrm{s}=0}=\left.\frac{1}{\mathrm{P}_{\mathrm{E}_{0 \mathrm{n}}}} \frac{\partial}{\partial \mathrm{s}}\left[\mathrm{W}_{\mathrm{E}_{0 \mathrm{n}}}(\mathrm{s})\right]\right|_{\mathrm{s}=0}
$$

Theorem: For the probability at each stage in the network, it must be in the range of $[0,1]$

Proof

$$
\text { Let } \mathrm{P}_{2}+\mathrm{P}_{3} \leq 1 \text {, where } \mathrm{P}_{2} \text { and } \mathrm{P}_{3} \geq 0 \text {, therefore } \mathrm{P}_{3} \leq\left(1-\mathrm{P}_{2}\right) \text {; }
$$




$$
\begin{aligned}
& \left(\left(1-\mathrm{P}_{4}\right)+\frac{\mathrm{P}_{4} \mathrm{P}_{6}}{\left(1-\mathrm{P}_{5}\right)}\right)=\frac{\left(1-\mathrm{P}_{5}\right)-\mathrm{P}_{4}\left(1-\mathrm{P}_{5}-\mathrm{P}_{6}\right)}{\left(1-\mathrm{P}_{5}\right)} \\
& \text { where } 0 \leq\left(1-\mathrm{P}_{5}-\mathrm{P}_{6}\right) \leq 1 \text {, and } 0 \leq \mathrm{P}_{4} \leq 1, \\
& \text { therefore } 0 \leq \mathrm{P}_{4}\left(1-\mathrm{P}_{5}-\mathrm{P}_{6}\right) \leq 1 ; \\
& \text { then }\left(1-\mathrm{P}_{5}\right)-\mathrm{P}_{4}\left(1-\mathrm{P}_{5}-\mathrm{P}_{6}\right) \leq\left(1-\mathrm{P}_{5}\right) ; \\
& \text { therefore } \frac{\mathrm{P}_{3}}{\left(1-\mathrm{P}_{2}\right)} \cdot\left(\left(1-\mathrm{P}_{4}\right)+\frac{\mathrm{P}_{4} \mathrm{P}_{6}}{\left(1-\mathrm{P}_{5}\right)}\right) \leq 1
\end{aligned}
$$

The expected time of product return is:

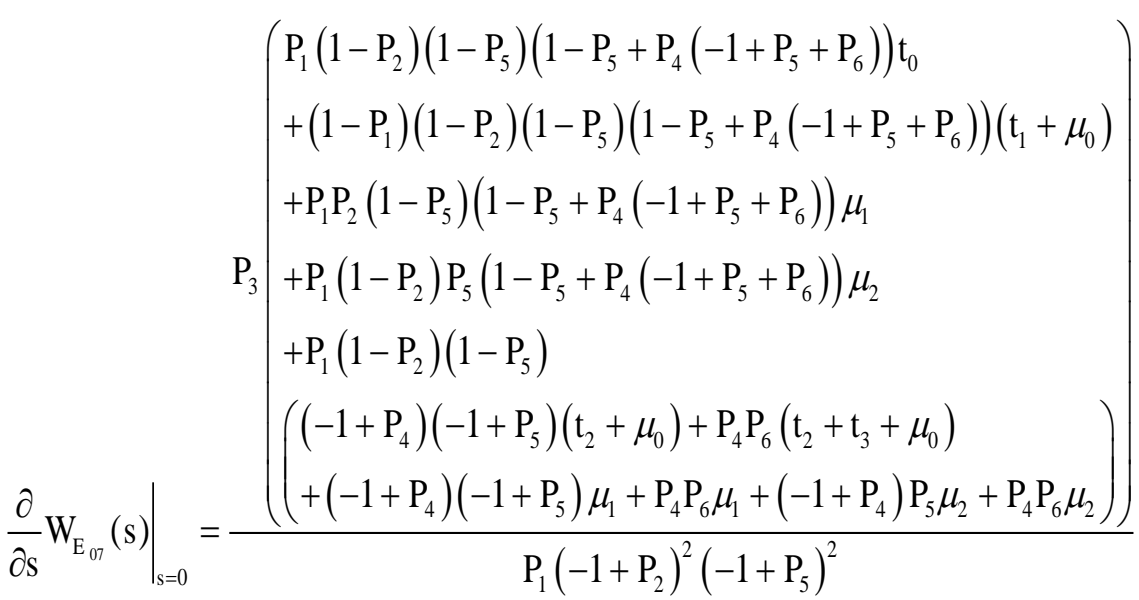

$$
\begin{aligned}
& \mathrm{t}_{\mathrm{E}_{07}}=\left.\frac{1}{\mathrm{P}_{\mathrm{E}_{07}}} \frac{\partial}{\partial \mathrm{s}}\left[\mathrm{W}_{\mathrm{E}_{07}}(\mathrm{~s})\right]\right|_{\mathrm{s}=0} \\
& =\left(\begin{array}{l}
\mathrm{t}_{0}+\left(\frac{1}{\mathrm{P}_{1}}-1\right) \mathrm{t}_{1}+\left(\frac{1}{\mathrm{P}_{1}}-1\right) \mu_{0}-\frac{\mathrm{P}_{2} \mu_{1}}{\mathrm{P}_{2}-1} \\
\left.+\frac{\left(\left(-1+\mathrm{P}_{5}\right)\left(\begin{array}{l}
\left(1-\mathrm{P}_{5}+\mathrm{P}_{4}\left(-1+\mathrm{P}_{5}+\mathrm{P}_{6}\right)\right) \mathrm{t}_{2}-\left(-1+\mathrm{P}_{5}\right)\left(\mu_{0}+\mu_{1}\right) \\
+\mathrm{P}_{4}\left(\left(-1+\mathrm{P}_{5}\right)\left(\mu_{0}+\mu_{1}\right)+\mathrm{P}_{6}\left(\mathrm{t}_{3}+\mu_{0}+\mu_{1}\right)\right)
\end{array}\right)-\mathrm{P}_{4} \mathrm{P}_{6} \mu_{2}\right.}{\left(\left(-1+\mathrm{P}_{5}\right)\left(1-\mathrm{P}_{5}+\mathrm{P}_{4}\left(-1+\mathrm{P}_{5}+\mathrm{P}_{6}\right)\right)\right)}\right)
\end{array}\right)
\end{aligned}
$$

\subsubsection{Forecasting remanufacture-able parts}

From node 7 to node 12, which is the parts inventory, the equivalence transfer function is

$$
\mathrm{W}_{\mathrm{E}_{7,12}}^{\mathrm{p}}(\mathrm{s})=\mathrm{W}_{78} \mathrm{~W}_{89} \mathrm{~W}_{9,12}^{\mathrm{p}}=\mathrm{e}^{\left(\mathrm{t}_{4}+\mathrm{t}_{5}+\mathrm{t}_{6}^{\mathrm{p}}\right) \mathrm{s}} \mathrm{P}_{7} \mathrm{P}_{9} \mathrm{P}_{11}^{\mathrm{p}}
$$

And the probability of remanufacturable parts $p$ is 


$$
\mathrm{P}_{\mathrm{E}_{7,12}}^{\mathrm{p}}=\mathrm{P}_{7} \mathrm{P}_{9} \mathrm{P}_{11}^{\mathrm{p}}
$$

Let $0 \leq \mathrm{P}_{7}, \mathrm{P}_{9}, \mathrm{P}_{11}^{\mathrm{p}} \leq 1 ; \quad$ We have $0 \leq \mathrm{P}_{7} \mathrm{P}_{9} \mathrm{P}_{11}^{\mathrm{p}} \leq 1$

The expected recycling time is

$$
\mathrm{t}_{\mathrm{E}_{7,12}}^{\mathrm{p}}=\mathrm{t}_{4}+\mathrm{t}_{5}+\mathrm{t}_{6}^{\mathrm{p}}
$$

If the quantity of returned product is $\mathrm{q}_{\mathrm{w}}$, the amount of $\mathrm{p}^{\text {th }}$ parts in each product's BOM is $q_{p}$, then the amount of remanufacturable $p^{\text {th }}$ part is

$$
\mathrm{Q}_{\mathrm{p}}=\left(\mathrm{P}_{\mathrm{E}_{07}} \mathrm{q}_{\mathrm{p}} \mathrm{P}_{\mathrm{E}_{7,12}}^{\mathrm{p}}\right) \cdot \mathrm{q}_{\mathrm{w}}=\mathrm{K}_{\mathrm{p}} \cdot \mathrm{q}_{\mathrm{w}}
$$

Substituting (11) and (17) into (20), we have

$$
K_{p}=\frac{P_{3} P_{7} P_{9} P_{11}^{p}}{\left(1-P_{2}\right)} \cdot\left(\left(1-P_{4}\right)+\frac{P_{4} P_{6}}{\left(1-P_{5}\right)}\right) \cdot q_{p}
$$

And the total remanufacturable part is

$$
\sum_{\mathrm{p}} \mathrm{Q}_{\mathrm{p}}=\left(\sum_{\mathrm{p}} \mathrm{K}_{\mathrm{p}}\right) \cdot \mathrm{q}_{\mathrm{w}}=\mathrm{K}_{\mathrm{parts}}^{*} \cdot \mathrm{q}_{\mathrm{w}}
$$

where $\mathrm{K}_{\text {parts }}^{*}$ means the remanufacturable parts in each product.

\subsubsection{Forecasting remanufacturable components}

Similarly, from node 7 to node 13 , which is the component inventory, the equivalence transfer function is

$$
\mathrm{W}_{\mathrm{E}_{7,13}}^{\mathrm{p}, \mathrm{c}}(\mathrm{s})=\mathrm{w}_{78}\left(\mathrm{w}_{89} \mathrm{w}_{9,10}^{\mathrm{p}}+\mathrm{w}_{8,10}\right) \mathrm{w}_{10,13}^{\mathrm{c}}=\mathrm{e}^{\left(\mathrm{t}_{4}+\mathrm{t}_{5}+\mathrm{t}_{7}^{\mathrm{c}}\right) \mathrm{s}} \mathrm{P}_{7} \mathrm{P}_{12}^{\mathrm{c}}\left(\mathrm{P}_{10}+\mathrm{e}^{\mathrm{t}_{6}^{\mathrm{p}} \mathrm{s}} \mathrm{P}_{9}\left(1-\mathrm{P}_{11}^{\mathrm{p}}\right)\right)
$$

and the probability of remanufacturable component $\mathrm{c}$ from part $\mathrm{p}$ with its recycling time expectation is 


$$
\mathrm{P}_{\mathrm{E}_{7,13}}^{\mathrm{p}, \mathrm{c}}=\mathrm{P}_{7} \mathrm{P}_{12}^{\mathrm{c}}\left(\mathrm{P}_{10}+\mathrm{P}_{9}\left(1-\mathrm{P}_{11}^{\mathrm{p}}\right)\right)
$$

Lemma 1: The probability from node 0 to 7 must be in the range of $[0,1]$.

Proof.

Let $0 \leq 1-\mathrm{P}_{11}^{\mathrm{p}} \leq 1$, we have $\mathrm{P}_{7} \mathrm{P}_{12}^{\mathrm{c}}\left(\mathrm{P}_{10}+\mathrm{P}_{9}\left(1-\mathrm{P}_{11}^{\mathrm{p}}\right)\right) \leq \mathrm{P}_{7} \mathrm{P}_{12}^{\mathrm{c}}\left(\mathrm{P}_{9}+\mathrm{P}_{10}\right)$;

We konw $0 \leq\left(\mathrm{P}_{9}+\mathrm{P}_{10}\right) \leq 1$ from node 8 , therefore

$$
\mathrm{P}_{7} \mathrm{P}_{12}^{\mathrm{c}}\left(\mathrm{P}_{10}+\mathrm{P}_{9}\left(1-\mathrm{P}_{11}^{\mathrm{p}}\right)\right) \leq \mathrm{P}_{7} \mathrm{P}_{12}^{\mathrm{c}}
$$

Because $0 \leq \mathrm{P}_{7} \leq 1,0 \leq \mathrm{P}_{12}^{\mathrm{c}} \leq 1$,

We have $0 \leq \mathrm{P}_{7} \mathrm{P}_{12}^{\mathrm{c}}\left(\mathrm{P}_{10}+\mathrm{P}_{9}\left(1-\mathrm{P}_{11}^{\mathrm{p}}\right)\right) \leq 1$

Therefore,

$$
\begin{aligned}
\left.\frac{\partial}{\partial s} \mathrm{~W}_{\mathrm{E}_{7,13}}^{\mathrm{p}, \mathrm{c}}(\mathrm{s})\right|_{\mathrm{s}=0}=\mathrm{P}_{7} \mathrm{P}_{12}^{\mathrm{c}}\left(\left(\mathrm{t}_{4}+\mathrm{t}_{5}+\mathrm{t}_{7}^{\mathrm{c}}\right)\left(\mathrm{P}_{10}+\mathrm{P}_{9}\left(1-\mathrm{P}_{11}^{\mathrm{p}}\right)\right)+\left(\mathrm{t}_{6}^{\mathrm{p}} \mathrm{P}_{9}\left(1-\mathrm{P}_{11}^{\mathrm{p}}\right)\right)\right. \\
\mathrm{t}_{\mathrm{E}_{7,13}^{\mathrm{p}, \mathrm{c}}(\mathrm{s})}=\frac{1}{\left.\mathrm{P}_{\mathrm{E}_{7,13}}^{\mathrm{p}, \mathrm{c}} \frac{\partial}{\partial s}\left[\mathrm{~W}_{\mathrm{E}_{7,13}}^{\mathrm{p}, \mathrm{c}}(\mathrm{s})\right]\right|_{\mathrm{s}=0}} \\
=\frac{\mathrm{P}_{7} \mathrm{P}_{12}^{\mathrm{c}}\left(\left(\mathrm{t}_{4}+\mathrm{t}_{5}+\mathrm{t}_{7}^{\mathrm{c}}\right)\left(\mathrm{P}_{10}+\mathrm{P}_{9}\left(1-\mathrm{P}_{11}^{\mathrm{p}}\right)\right)+\left(\mathrm{t}_{6}^{\mathrm{p}} \mathrm{P}_{9}\left(1-\mathrm{P}_{11}^{\mathrm{p}}\right)\right)\right.}{\mathrm{P}_{7} \mathrm{P}_{12}^{\mathrm{c}}\left(\mathrm{P}_{10}+\mathrm{P}_{9}\left(1-\mathrm{P}_{11}^{\mathrm{p}}\right)\right)} \\
=\frac{\mathrm{P}_{10}\left(\mathrm{t}_{4}+\mathrm{t}_{5}+\mathrm{t}_{7}^{\mathrm{c}}\right)+\mathrm{P}_{9}\left(1-\mathrm{P}_{11}^{\mathrm{p}}\right)\left(\mathrm{t}_{4}+\mathrm{t}_{5}+\mathrm{t}_{6}^{\mathrm{p}}+\mathrm{t}_{7}^{\mathrm{c}}\right)}{\mathrm{P}_{10}+\mathrm{P}_{9}-\mathrm{P}_{11}^{\mathrm{p}} \mathrm{P}_{9}} \\
=\mathrm{t}_{4}+\mathrm{t}_{5}+\mathrm{t}_{6}^{\mathrm{p}}+\mathrm{t}_{7}^{\mathrm{c}}-\frac{\mathrm{P}_{10} \mathrm{t}_{6}^{\mathrm{p}}}{\mathrm{P}_{10}+\mathrm{P}_{9}\left(1-\mathrm{P}_{11}^{\mathrm{p}}\right)}
\end{aligned}
$$

If the quantity of product is $\mathrm{q}_{\mathrm{w}}$, the amount of the $\mathrm{c}^{\text {th }}$ component from the $\mathrm{p}^{\text {th }}$ part in each product is $q_{c}^{p}$, then the amount of the returned $c^{\text {th }}$ component from the $\mathrm{p}^{\text {th }}$ part is

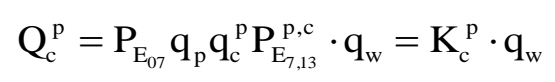

where 


$$
\mathrm{K}_{\mathrm{c}}^{\mathrm{p}}=\frac{\mathrm{P}_{3}}{\left(1-\mathrm{P}_{2}\right)} \cdot\left(\left(1-\mathrm{P}_{4}\right)+\frac{\mathrm{P}_{4} \mathrm{P}_{6}}{\left(1-\mathrm{P}_{5}\right)}\right) \mathrm{P}_{7} \mathrm{P}_{12}^{\mathrm{c}}\left(\mathrm{P}_{10}+\mathrm{P}_{9}\left(1-\mathrm{P}_{11}^{\mathrm{p}}\right)\right) \mathrm{q}_{\mathrm{p}} \mathrm{q}_{\mathrm{c}}^{\mathrm{p}}
$$

The total returned component is

$$
\sum_{\mathrm{c}} \sum_{\mathrm{p}} \mathrm{Q}_{\mathrm{c}}^{\mathrm{p}}=\left(\sum_{\mathrm{c}} \sum_{\mathrm{p}} \mathrm{K}_{\mathrm{c}}^{\mathrm{p}}\right) \cdot \mathrm{q}_{\mathrm{w}}=\mathrm{K}_{\text {components }}^{*} \cdot \mathrm{q}_{\mathrm{w}}
$$

where $\mathrm{K}_{\text {components }}^{*}$ is the total remanufacturable component in each product.

\subsubsection{Forecasting recyclable materials}

Likewise, from node 7 to node 14 , which is the material inventory, the equivalence transfer function is

$$
\begin{aligned}
& \mathrm{W}_{\mathrm{E}_{7,14}^{\mathrm{p}, \mathrm{c} m}}(\mathrm{~s})=\left(\mathrm{w}_{7,11}+\mathrm{w}_{78}\left(\mathrm{w}_{8,11}+\left(\mathrm{w}_{8,10}+\mathrm{w}_{89} \mathrm{w}_{9,10}^{\mathrm{p}}\right) \mathrm{w}_{10,11}^{\mathrm{c}}\right)\right) \mathrm{w}_{11,14}^{\mathrm{m}} \\
& =\mathrm{e}^{\left.\mathrm{s} \mathrm{t}_{4}+\mathrm{t}_{8}^{\mathrm{m}}\right)}\left(\mathrm{P}_{8}+\mathrm{P}_{7} \mathrm{e}^{\mathrm{st} \mathrm{t}_{5}}\left(\left(1-\mathrm{P}_{9}-\mathrm{P}_{10}\right)+\left(\mathrm{P}_{10}+\mathrm{P}_{9}\left(1-\mathrm{P}_{11}^{\mathrm{p}}\right) \mathrm{e}^{\mathrm{st}_{6}^{\mathrm{p}}}\right)\left(1-\mathrm{P}_{12}^{\mathrm{c}}\right) \mathrm{e}^{\mathrm{st}_{7}^{\mathrm{c}}}\right)\right) \mathrm{P}_{13}^{\mathrm{m}}
\end{aligned}
$$

The probability and time of material recycle for component $i$ are

$$
\begin{aligned}
\mathrm{P}_{\mathrm{E}_{7,14}}^{\mathrm{p}, \mathrm{c}, \mathrm{m}} & =\left(\mathrm{P}_{8}+\mathrm{P}_{7}\left(\left(1-\mathrm{P}_{9}-\mathrm{P}_{10}\right)+\left(\mathrm{P}_{10}+\mathrm{P}_{9}\left(1-\mathrm{P}_{11}^{\mathrm{p}}\right)\right)\left(1-\mathrm{P}_{12}^{\mathrm{c}}\right)\right)\right) \mathrm{P}_{13}^{\mathrm{m}} \\
& =\left(\mathrm{P}_{8}+\mathrm{P}_{7}\left(1-\left(\mathrm{P}_{9}+\mathrm{P}_{10}\right) \mathrm{P}_{12}^{\mathrm{c}}-\mathrm{P}_{9} \mathrm{P}_{11}^{\mathrm{p}}\left(1-\mathrm{P}_{12}^{\mathrm{c}}\right)\right)\right) \mathrm{P}_{13}^{\mathrm{m}} \\
& =\left(\mathrm{P}_{8}+\mathrm{P}_{7}\left(1+\mathrm{P}_{9} \mathrm{P}_{11}^{\mathrm{p}}\left(\mathrm{P}_{12}^{\mathrm{c}}-1\right)-\mathrm{P}_{12}^{\mathrm{c}}\left(\mathrm{P}_{10}+\mathrm{P}_{9}\right)\right)\right) \mathrm{P}_{13}^{\mathrm{m}}
\end{aligned}
$$

Lemma 2: The probability from node 7 to 14 must be in the range of $[0,1]$.

Proof.

$$
\begin{aligned}
& \text { For } \mathrm{P}_{\mathrm{E}_{7,14}}^{\mathrm{p}, \mathrm{c}, \mathrm{m}}=\left(\mathrm{P}_{8}+\mathrm{P}_{7}\left(\left(1-\mathrm{P}_{9}-\mathrm{P}_{10}\right)+\left(\mathrm{P}_{10}+\mathrm{P}_{9}\left(1-\mathrm{P}_{11}^{\mathrm{p}}\right)\right)\left(1-\mathrm{P}_{12}^{\mathrm{c}}\right)\right)\right) \mathrm{P}_{13}^{\mathrm{m}} \\
& \text { Because } 1-\mathrm{P}_{9}-\mathrm{P}_{10} \geq 0,\left(1-\mathrm{P}_{11}^{\mathrm{p}}\right) \geq 0,\left(1-\mathrm{P}_{12}^{\mathrm{c}}\right) \geq 0 ; \mathrm{P}_{8}, \mathrm{P}_{7}, \mathrm{P}_{10}, \mathrm{P}_{9}, \mathrm{P}_{13}^{\mathrm{m}} \geq 0 \\
& \text { Therefore } \quad \mathrm{P}_{\mathrm{E}_{7,14}^{\mathrm{p}, \mathrm{c}, \mathrm{m}} \geq 0}
\end{aligned}
$$


Because $0 \leq\left(\mathrm{P}_{9}+\mathrm{P}_{10}\right) \leq 1$

We have $\mathrm{P}_{\mathrm{E}_{7,14}}^{\mathrm{p}, \mathrm{m}} \leq\left(\mathrm{P}_{8}+\mathrm{P}_{7}\left(1-\mathrm{P}_{9} \mathrm{P}_{11}^{\mathrm{p}}\left(1-\mathrm{P}_{12}^{\mathrm{c}}\right)\right)\right) \mathrm{P}_{13}^{\mathrm{m}}$;

Also, because $\mathrm{P}_{9}, \mathrm{P}_{11}^{\mathrm{p}} \geq 0$, and $\left(1-\mathrm{P}_{12}^{\mathrm{c}}\right) \geq 0$,

We have $\mathrm{P}_{\mathrm{E}_{7,14}}^{\mathrm{p}, \mathrm{m}} \leq\left(\mathrm{P}_{8}+\mathrm{P}_{7}\right) \mathrm{P}_{13}^{\mathrm{m}}$;

And $0 \leq \mathrm{P}_{7}, \mathrm{P}_{8}, \mathrm{P}_{13}^{\mathrm{m}} \leq 1$,

Therefore, $\quad \mathrm{P}_{\mathrm{E}_{7,14}}^{\mathrm{p}, \mathrm{c}, \mathrm{m}} \leq 1$

Hence, we have

$$
\left.\frac{\partial}{\partial \mathrm{s}} \mathrm{W}_{\mathrm{F}_{7,14} \mathrm{p}, \mathrm{m}}(\mathrm{s})\right|_{\mathrm{s}=0}=\mathrm{P}_{13}^{\mathrm{m}}\left(\begin{array}{l}
\mathrm{P}_{8}\left(\mathrm{t}_{4}+\mathrm{t}_{8}^{\mathrm{m}}\right)-\mathrm{P}_{7}\left(-1+\mathrm{P}_{10}+\mathrm{P}_{9}\right)\left(\mathrm{t}_{4}+\mathrm{t}_{5}+\mathrm{t}_{8}^{\mathrm{m}}\right) \\
-\mathrm{P}_{10}\left(\mathrm{P}_{12}^{\mathrm{c}}-1\right) \cdot \mathrm{P}_{7}\left(\mathrm{t}_{4}+\mathrm{t}_{5}+\mathrm{t}_{7}^{\mathrm{c}}+\mathrm{t}_{8}^{\mathrm{m}}\right)+ \\
\left(\mathrm{P}_{11}^{\mathrm{p}}-1\right)\left(\mathrm{P}_{12}^{\mathrm{c}}-1\right) \mathrm{P}_{7} \mathrm{P}_{9}\left(\mathrm{t}_{4}+\mathrm{t}_{5}+\mathrm{t}_{6}^{\mathrm{p}}+\mathrm{t}_{7}^{\mathrm{c}}+\mathrm{t}_{8}^{\mathrm{m}}\right)
\end{array}\right)
$$

$$
\begin{aligned}
& \mathfrak{t}_{\mathrm{E}_{7,14}}^{\mathrm{p}, \mathrm{c}, \mathrm{m}}(\mathrm{s})=\left.\frac{1}{\mathrm{P}_{\mathrm{E}_{7,14}}^{\mathrm{p}, \mathrm{c}, \mathrm{m}}} \frac{\partial}{\partial \mathrm{s}}\left[\mathrm{W}_{\mathrm{E}_{7,14}}^{\mathrm{p}, \mathrm{c}, \mathrm{m}}(\mathrm{s})\right]\right|_{\mathrm{s}=0} \\
& =\frac{\left(\begin{array}{l}
\mathrm{P}_{8}\left(\mathrm{t}_{4}+\mathrm{t}_{8}^{\mathrm{m}}\right)-\mathrm{P}_{7}\left(\mathrm{P}_{10}+\mathrm{P}_{9}-1\right)\left(\mathrm{t}_{4}+\mathrm{t}_{5}+\mathrm{t}_{8}^{\mathrm{m}}\right)- \\
\mathrm{P}_{10}\left(\mathrm{p}_{12}^{\mathrm{c}}-1\right) \mathrm{P}_{7} \cdot\left(\mathrm{t}_{4}+\mathrm{t}_{5}+\mathrm{t}_{7}^{\mathrm{c}}+\mathrm{t}_{8}^{\mathrm{m}}\right)+\mathrm{P}\left(\mathrm{P}_{11}^{\mathrm{p}}-1\right) \cdot \\
\left(\mathrm{P}_{12}^{\mathrm{c}}-1\right) \mathrm{P}_{7} \mathrm{P}_{9}\left(\mathrm{t}_{4}+\mathrm{t}_{5}+\mathrm{t}_{6}^{\mathrm{p}}+\mathrm{t}_{7}^{\mathrm{c}}+\mathrm{t}_{8}^{\mathrm{m}}\right)
\end{array}\right)}{\mathrm{P}_{8}+\mathrm{P}_{7}\left(1+\mathrm{P}_{9} \mathrm{P}_{11}^{\mathrm{p}}\left(\mathrm{P}_{12}^{\mathrm{c}}-1\right)-\mathrm{P}_{12}^{\mathrm{c}}\left(\mathrm{P}_{10}+\mathrm{P}_{9}\right)\right)} \\
& =\frac{\left(\mathrm{P}_{8}\left(\mathrm{t}_{4}+\mathrm{t}_{8}^{\mathrm{m}}\right)+\mathrm{P}_{7}\left(\begin{array}{l}
\left(1-\mathrm{P}_{10}-\mathrm{P}_{9}\right)\left(\mathrm{t}_{4}+\mathrm{t}_{5}+\mathrm{t}_{8}^{\mathrm{m}}\right)-\mathrm{P}_{10}\left(\mathrm{p}_{12}^{\mathrm{c}}-1\right) \\
\left(\mathrm{t}_{4}+\mathrm{t}_{5}+\mathrm{t}_{7}^{\mathrm{c}}+\mathrm{t}_{8}^{\mathrm{m}}\right)+\left(\mathrm{P}_{11}^{\mathrm{p}}-1\right)\left(\mathrm{P}_{12}^{\mathrm{c}}-1\right) \mathrm{P}_{9} \cdot \\
\left(\mathrm{t}_{4}+\mathrm{t}_{5}+\mathrm{t}_{6}^{\mathrm{p}}+\mathrm{t}_{7}^{\mathrm{c}}+\mathrm{t}_{8}^{\mathrm{m}}\right)
\end{array}\right)\right.}{\mathrm{P}_{8}+\mathrm{P}_{7}\left(1+\mathrm{P}_{9} \mathrm{P}_{11}^{\mathrm{p}}\left(\mathrm{P}_{12}^{\mathrm{c}}-1\right)-\mathrm{P}_{12}^{\mathrm{c}}\left(\mathrm{P}_{10}+\mathrm{P}_{9}\right)\right)} \\
& =\mathrm{t}_{4}+\mathrm{t}_{5}+\mathrm{t}_{6}^{\mathrm{p}}+\mathrm{t}_{7}^{\mathrm{c}}+\mathrm{t}_{8}^{\mathrm{m}}-\frac{\left(\mathrm{t}_{5}+\mathrm{t}_{6}^{\mathrm{p}}+\mathrm{t}_{7}^{\mathrm{c}}\right) \mathrm{P}_{8}+\mathrm{P}_{7}\left(\begin{array}{c}
\left(1-\mathrm{P}_{9}\right)\left(\mathrm{t}_{6}^{\mathrm{p}}+\mathrm{t}_{7}^{\mathrm{c}}\right) \\
-\mathrm{P}_{10}\left(\mathrm{t}_{7}^{\mathrm{c}}+\mathrm{P}_{12}^{\mathrm{c}} \mathrm{t}_{6}^{\mathrm{p}}\right)
\end{array}\right)}{\mathrm{P}_{8}+\mathrm{P}_{7}\left(1+\mathrm{P}_{9} \mathrm{P}_{11}^{\mathrm{p}}\left(\mathrm{P}_{12}^{\mathrm{c}}-1\right)-\mathrm{P}_{12}^{\mathrm{c}}\left(\mathrm{P}_{10}+\mathrm{P}_{9}\right)\right)}
\end{aligned}
$$

If the weight of material $\mathrm{m}$ from component $\mathrm{c}$ is $\mathrm{g}_{\mathrm{m}}^{\mathrm{c}}$, the returned weight of $\mathrm{m}$ is

$$
\mathrm{M}_{\mathrm{m}}^{\mathrm{p}, \mathrm{c}}=\mathrm{P}_{\mathrm{E}_{07}} \mathrm{q}_{\mathrm{p}} \mathrm{q}_{\mathrm{c}}^{\mathrm{p}} \mathrm{P}_{\mathrm{E}_{7,14}} \mathrm{~g}_{\mathrm{m}}^{\mathrm{c}} \cdot \mathrm{q}_{\mathrm{w}}=\mathrm{K}_{\mathrm{m}}^{\mathrm{p}, \mathrm{c}} \cdot \mathrm{q}_{\mathrm{w}}
$$


where

$$
\begin{aligned}
& \mathrm{K}_{\mathrm{m}}^{\mathrm{p}, \mathrm{c}}=\frac{\mathrm{P}_{3}}{\left(1-\mathrm{P}_{2}\right)} \cdot\left(\left(1-\mathrm{P}_{4}\right)+\frac{\mathrm{P}_{4} \mathrm{P}_{6}}{\left(1-\mathrm{P}_{5}\right)}\right) \\
& \left(\left(\mathrm{P}_{8}+\mathrm{P}_{7}\left(1-\left(\mathrm{P}_{9}+\mathrm{P}_{10}\right) \mathrm{P}_{12}^{\mathrm{c}}-\mathrm{P}_{9} \mathrm{P}_{11}^{\mathrm{p}}\left(1-\mathrm{P}_{12}^{\mathrm{c}}\right)\right)\right) \mathrm{P}_{13}^{\mathrm{m}}\right) \mathrm{q}_{\mathrm{p}} \cdot \mathrm{q}_{\mathrm{c}}^{\mathrm{p}} \cdot \mathrm{g}_{\mathrm{m}}^{\mathrm{c}}
\end{aligned}
$$

And the total weight of the returned component is

$$
\sum_{\mathrm{m}} \sum_{\mathrm{c}} \sum_{\mathrm{p}} \mathrm{M}_{\mathrm{m}}^{\mathrm{p}, \mathrm{c}}=\left(\sum_{\mathrm{m}} \sum_{\mathrm{c}} \sum_{\mathrm{p}} \mathrm{K}_{\mathrm{m}}^{\mathrm{p}, \mathrm{c}}\right) \cdot \mathrm{q}_{\mathrm{w}}=\mathrm{K}_{\text {material }}^{*} \cdot \mathrm{q}_{\mathrm{w}}
$$

where $\mathrm{K}_{\text {material }}^{*}$ is the total renewable material in each product.

\subsubsection{Forecasting discarded materials}

Finally, from node 7 to node 15 , which is the material inventory, the equivalence transfer function is

$$
\begin{aligned}
\mathrm{W}_{\mathrm{E}_{7,15}}^{\mathrm{p}, \mathrm{c}, \mathrm{m}}(\mathrm{s})= & \left(\mathrm{w}_{7,11}+\mathrm{W}_{78}\left(\mathrm{w}_{8,11}+\left(\mathrm{w}_{8,10}+\mathrm{w}_{89} \mathrm{~W}_{9,10}^{\mathrm{p}}\right) \mathrm{w}_{10,11}^{\mathrm{c}}\right)\right) \mathrm{w}_{11,15}^{\mathrm{m}}+\mathrm{w}_{7,15} \\
= & \mathrm{e}^{\mathrm{s}\left(\mathrm{t}_{4}+\mathrm{t}_{8}^{\mathrm{m}}\right)}\left(\mathrm{P}_{8}+\mathrm{P}_{7} \mathrm{e}^{\mathrm{st}_{5}}\left(\begin{array}{l}
\left(1-\mathrm{P}_{9}-\mathrm{P}_{10}\right) \\
+\left(\mathrm{P}_{10}+\mathrm{P}_{9}\left(1-\mathrm{P}_{11}^{\mathrm{p}}\right) \mathrm{e}^{\mathrm{st}_{6}^{\mathrm{p}}}\right)\left(1-\mathrm{P}_{12}^{\mathrm{c}}\right) \mathrm{e}^{\mathrm{st} \mathrm{c}}
\end{array}\right)\right)\left(1-\mathrm{P}_{13}^{\mathrm{m}}\right) \\
& +\left(1-\mathrm{P}_{7}-\mathrm{P}_{8}\right) \mathrm{e}^{\mathrm{t}_{4} \mathrm{~s}}
\end{aligned}
$$

The probability and time of raw material manufacture for component $\mathrm{c}$ are

$$
\begin{aligned}
\mathrm{E}_{\mathrm{E}_{7,15}}^{\mathrm{p}, \mathrm{c}, \mathrm{m}} & =\left(\mathrm{P}_{8}+\mathrm{P}_{7}\left(\left(1-\mathrm{P}_{9}-\mathrm{P}_{10}\right)+\left(\mathrm{P}_{10}+\mathrm{P}_{9}\left(1-\mathrm{P}_{11}^{\mathrm{p}}\right)\right)\left(1-\mathrm{P}_{12}^{\mathrm{c}}\right)\right)\right)\left(1-\mathrm{P}_{13}^{\mathrm{m}}\right) \\
& +\left(1-\mathrm{P}_{7}-\mathrm{P}_{8}\right) \\
& =\left(\mathrm{P}_{8}+\mathrm{P}_{7}\left(1-\left(\mathrm{P}_{9}+\mathrm{P}_{10}\right) \mathrm{P}_{12}^{\mathrm{c}}-\mathrm{P}_{9} \mathrm{P}_{11}^{\mathrm{p}}\left(1-\mathrm{P}_{12}^{\mathrm{c}}\right)\right)\right)\left(1-\mathrm{P}_{13}^{\mathrm{m}}\right)+\left(1-\mathrm{P}_{7}-\mathrm{P}_{8}\right)
\end{aligned}
$$

Lemma 3: The probability from node 7 to 15 must be in the range of $[0,1]$.

Proof. 
Because $1-\mathrm{P}_{9}-\mathrm{P}_{10} \geq 0,1-\mathrm{P}_{7}-\mathrm{P}_{8} \geq 0,\left(1-\mathrm{P}_{11}^{\mathrm{p}}\right) \geq 0$,

$$
\left(1-P_{12}^{c}\right) \geq 0,\left(1-P_{13}^{m}\right) \geq 0 ; \text { and } P_{8}, P_{7}, P_{10}, P_{9}, P_{13}^{m} \geq 0
$$

Therefore, $\quad \mathrm{P}_{\mathrm{E}_{7} 15}^{\mathrm{p}, \mathrm{cm}} \geq 0$

$$
\text { For } \begin{aligned}
\mathrm{P}_{\mathrm{F}_{7,15}}^{\mathrm{p}, \mathrm{c}, \mathrm{m}}= & \left(\mathrm{P}_{8}+\mathrm{P}_{7}\left(1-\left(\mathrm{P}_{9}+\mathrm{P}_{10}\right) \mathrm{P}_{12}^{\mathrm{c}}-\mathrm{P}_{9} \mathrm{P}_{11}^{\mathrm{p}}\left(1-\mathrm{P}_{12}^{\mathrm{c}}\right)\right)\right)\left(1-\mathrm{P}_{13}^{\mathrm{m}}\right) \\
& +\left(1-\mathrm{P}_{7}-\mathrm{P}_{8}\right)
\end{aligned}
$$

Because, $0 \leq\left(\mathrm{P}_{9}+\mathrm{P}_{10}\right) \leq 1$,

$$
\text { Hence, } \mathrm{P}_{\mathrm{E}_{7,15}}^{\mathrm{p}, \mathrm{c}, \mathrm{m}} \leq\left(\mathrm{P}_{8}+\mathrm{P}_{7}\left(1-\mathrm{P}_{9} \mathrm{P}_{11}^{\mathrm{p}}\left(1-\mathrm{P}_{12}^{\mathrm{c}}\right)\right)\right)\left(1-\mathrm{P}_{13}^{\mathrm{m}}\right)+\left(1-\mathrm{P}_{7}-\mathrm{P}_{8}\right) \text {; }
$$

also

$$
\begin{aligned}
& \text { because } \mathrm{P}_{9}, \mathrm{P}_{11}^{\mathrm{p}} \geq 0 \text {, and }\left(1-\mathrm{P}_{12}^{\mathrm{c}}\right) \geq 0 \text {, } \\
& \text { then } \mathrm{P}_{\mathrm{F}_{7,15}}^{\mathrm{p}, \mathrm{cm}} \leq\left(\mathrm{P}_{8}+\mathrm{P}_{7}\right)\left(1-\mathrm{P}_{13}^{\mathrm{m}}\right)+\left(1-\mathrm{P}_{7}-\mathrm{P}_{8}\right) \text {; } \\
& \text { and because } 0 \leq \mathrm{P}_{7}, \mathrm{P}_{8},\left(1-\mathrm{P}_{13}^{\mathrm{m}}\right) \leq 1 \text {, }
\end{aligned}
$$

$$
\left(\mathrm{P}_{8}+\mathrm{P}_{7}\right)\left(1-\mathrm{P}_{13}^{\mathrm{m}}\right)+\left(1-\mathrm{P}_{7}-\mathrm{P}_{8}\right)=1-\left(\mathrm{P}_{8}+\mathrm{P}_{7}\right) \mathrm{P}_{13}^{\mathrm{m}}
$$

Therefore, $\mathrm{P}_{\mathrm{E}_{7}, 15}^{\mathrm{p}, \mathrm{m}} \leq 1$

Hence, we have

$$
\begin{aligned}
& \left.\frac{\partial}{\partial \mathrm{s}} \mathrm{W}_{\mathrm{F}_{7,15}, \mathrm{p}, \mathrm{m}}(\mathrm{s})\right|_{\mathrm{s}=0}=-\left(\mathrm{P}_{7}+\mathrm{P}_{8}-1\right) \mathrm{t}_{4}-\left(\mathrm{P}_{13}^{\mathrm{m}}-1\right) \mathrm{P}_{8}\left(\mathrm{t}_{4}+\mathrm{t}_{8}^{\mathrm{m}}\right) \\
& +\left(\mathrm{P}_{13}^{\mathrm{m}}-1\right) \mathrm{P}_{7}\left(\mathrm{P}_{10}+\mathrm{P}_{9}-1\right)\left(\mathrm{t}_{4}+\mathrm{t}_{5}+\mathrm{t}_{8}^{\mathrm{m}}\right) \\
& +\mathrm{P}_{7} \mathrm{P}_{10}\left(\mathrm{P}_{12}^{\mathrm{c}}-1\right)\left(\mathrm{P}_{13}^{\mathrm{m}}-1\right)\left(\mathrm{t}_{4}+\mathrm{t}_{5}+\mathrm{t}_{7}^{\mathrm{c}}+\mathrm{t}_{8}^{\mathrm{m}}\right) \\
& -\mathrm{P}_{7} \mathrm{P}_{9}\left(\mathrm{P}_{11}^{\mathrm{p}}-1\right)\left(\mathrm{P}_{12}^{\mathrm{c}}-1\right)\left(\mathrm{P}_{13}^{\mathrm{m}}-1\right)\left(\mathrm{t}_{4}+\mathrm{t}_{5}+\mathrm{t}_{6}^{\mathrm{p}}+\mathrm{t}_{7}^{\mathrm{c}}+\mathrm{t}_{8}^{\mathrm{m}}\right) \\
& \mathfrak{t}_{\mathrm{E}_{7,15}}^{\mathrm{p}, \mathrm{c}, \mathrm{m}}(\mathrm{s})=\left.\frac{1}{\mathrm{P}_{\mathrm{F}_{715}}^{\mathrm{p}, \mathrm{c}, \mathrm{m}}} \frac{\partial}{\partial \mathrm{s}}\left[\mathrm{W}_{\mathrm{E}_{7,15}}^{\mathrm{p}, \mathrm{c}, \mathrm{m}}(\mathrm{s})\right]\right|_{\mathrm{s}=0} \\
& =\frac{\left(\begin{array}{l}
-\left(\mathrm{P}_{7}+\mathrm{P}_{8}-1\right) \mathrm{t}_{4}-\left(\mathrm{P}_{13}^{\mathrm{m}}-1\right) \mathrm{P}_{8}\left(\mathrm{t}_{4}+\mathrm{t}_{8}^{\mathrm{m}}\right) \\
+\left(\mathrm{P}_{13}^{\mathrm{m}}-1\right) \mathrm{P}_{7}\left(\mathrm{P}_{10}+\mathrm{P}_{9}-1\right)\left(\mathrm{t}_{4}+\mathrm{t}_{5}+\mathrm{t}_{8}^{\mathrm{m}}\right) \\
+\mathrm{P}_{7} \mathrm{P}_{10}\left(\mathrm{P}_{12}^{\mathrm{c}}-1\right)\left(\mathrm{P}_{13}^{\mathrm{m}}-1\right)\left(\mathrm{t}_{4}+\mathrm{t}_{5}+\mathrm{t}_{7}^{\mathrm{c}}+\mathrm{t}_{8}^{\mathrm{m}}\right) \\
-\mathrm{P}_{7} \mathrm{P}_{9}\left(\mathrm{P}_{11}^{\mathrm{p}}-1\right)\left(\mathrm{P}_{12}^{\mathrm{c}}-1\right)\left(\mathrm{P}_{13}^{\mathrm{m}}-1\right)\left(\mathrm{t}_{4}+\mathrm{t}_{5}+\mathrm{t}_{6}^{\mathrm{p}}+\mathrm{t}_{7}^{\mathrm{c}}+\mathrm{t}_{8}^{\mathrm{m}}\right)
\end{array}\right)}{\left(\left(\mathrm{P}_{8}+\mathrm{P}_{7}\left(1-\left(\mathrm{P}_{9}+\mathrm{P}_{10}\right) \mathrm{P}_{12}^{\mathrm{c}}-\mathrm{P}_{9} \mathrm{P}_{11}^{\mathrm{p}}\left(1-\mathrm{P}_{12}^{\mathrm{c}}\right)\right)\right)\left(1-\mathrm{P}_{13}^{\mathrm{m}}\right)+\left(1-\mathrm{P}_{7}-\mathrm{P}_{8}\right)\right)}
\end{aligned}
$$

If the weight of discarded material $m$ from component $j$ is $\mathrm{d}_{\mathrm{m}}^{\mathrm{c}}$, the discarded weight of $m$ is 


$$
\mathrm{D}_{\mathrm{m}}^{\mathrm{p}, \mathrm{c}}=\mathrm{P}_{\mathrm{E}_{07}} \mathrm{q}_{\mathrm{p}} \mathrm{q}_{\mathrm{c}}^{\mathrm{p}} \mathrm{P}_{\mathrm{E}_{7,15}} \mathrm{~d}_{\mathrm{m}}^{\mathrm{c}} \cdot \mathrm{q}_{\mathrm{w}}=\mathrm{K}_{\mathrm{d}}^{\mathrm{p}, \mathrm{c}, \mathrm{m}} \cdot \mathrm{q}_{\mathrm{w}}
$$

where

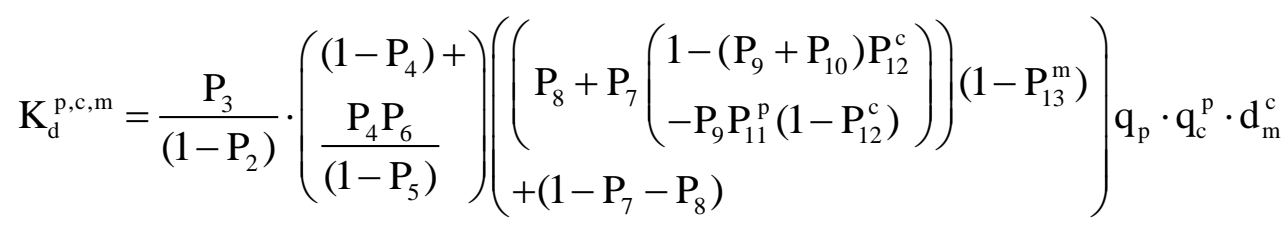

And the total weight of the returned component is

$$
\sum_{\mathrm{m}} \sum_{\mathrm{c}} \sum_{\mathrm{p}} \mathrm{D}_{\mathrm{m}}^{\mathrm{p}, \mathrm{c}}=\left(\sum_{\mathrm{m}} \sum_{\mathrm{c}} \sum_{\mathrm{p}} \mathrm{K}_{\mathrm{d}}^{\mathrm{p}, \mathrm{c}, \mathrm{m}}\right) \cdot \mathrm{q}_{\mathrm{w}}=\mathrm{K}_{\mathrm{discard}}^{*} \cdot \mathrm{q}_{\mathrm{w}}
$$

where $\mathrm{K}_{\mathrm{discard}}^{*}$ is the total discarded material in each product.

\section{Numerical example}

A printer manufacturer begins to deal with product return and remanufacturing. Table 4 shows the probability and time duration of a printer's return and recycling. One printer can be disassembled into four parts: ink cartridge, cleaning device, trolley and paper feeder. Each part contains screws, chips, plastic components and metal components (or some of them). In line with Insert Figure 2, the parameters are listed in Tables 4 and 5.

Insert Table 4,5,6 and 7 here.

(1) Prediction of product returns:

Applying Eq. (12) and Eq.(14)-(15) and Table 4, the probability of the product returning is $\mathrm{P}_{\mathrm{E}_{07}}=0.82$, and the expected return time is $\mathrm{t}_{\mathrm{E}_{07}}=200.9$ weeks.

Insert Figure 3 here. 
Although the mean $\mu$ and variance $\sigma^{2}=\left.\frac{\partial^{2}}{\partial \mathrm{s}^{2}}\left[\mathrm{M}_{\mathrm{E}_{0 \mathrm{n}}}(\mathrm{s})\right]\right|_{\mathrm{s}=0}-\mu^{2}$ can be derived, it is not possible to decide which distribution exactly is. To verify the analytic result, we cross-checked it through a simulation in Matlab. The results of product return probability density function (PDF) is shown in Figure 3 and the cumulative probability function $(\mathrm{CPF})$ in Figure 4 . Figure 3 indicates that the expected product return peak time is week 200.94 with the probability of $82.04 \%$, which matches the analytic result. In addition, the PDF shows a negative skewness. It means after the expected time, product return rate will rapidly reduce. This is useful information for remanufacturers when planning capacity in advance. From Figure 4, it suggests that the remanufacturer should consider allocating the major resources no later than week 150 when the return rate reaches $50 \%$, for the sake of economic benefits.

(2) Prediction of renewable parts

Applying Eq. (17) and (19), the renewable parts probability and expected time are derived. For example, the probability and expected time for the ink cartridge are $\mathrm{P}_{\mathrm{E}_{7,12}}^{1}=0.39, \mathrm{t}_{\mathrm{E}_{7,12}}^{1}=0.65$ weeks.

\section{(3) Prediction of remanufactured components}

Remanufactured components can be predicted by using Eq. (24), (26) and (27). For example, the probability and expected time for the chip from the paper feeder are $\mathrm{P}_{\mathrm{E}_{7,13}}^{2,4}=0.2176, \mathrm{t}_{\mathrm{E}_{7,13}}^{2,4}=1.1735$ weeks.

(4) Prediction of extracted materials 
For example, through Eq. (32), (34) and (35), the probability and expected time for remanufactured raw plastic from plastic parts on the trolley are $\mathrm{P}_{\mathrm{E}_{7,14}^{3,1}}^{3,1}=0.32$, $\mathrm{t}_{\mathrm{E}_{7,14}}^{3,3,1}=0.9350$ weeks.

(5) Prediction of disposal waste

Equations Eq. (40), (42) and (43) are used to calculate the discarded waste. For example, the probability and expected time for discarded metal from metal parts on the cleaning device are $\mathrm{P}_{\mathrm{E}_{7,15}}^{2,4,2}=0.09, \mathrm{t}_{\mathrm{E}_{7,15}}^{2,4,2}=0.6341$ weeks.

For 1000 printers, the renewed, remanufactured, recycled and discarded materials are as follows:

(1) Through Eq. (20)-(22), 1054 parts will be remanufactured, including 321 ink cartridges, 137 cleaning devices, 229 trolleys and 367 paper feeders.

(2) There are 9664 remanufactured components, including 6335 screws, 467 chips, 1378 plastic components and 1484 metal components, by using Eq. (28)-(30).

(3) Applying Eq. (36)-(38), total recycled materials are $1827.4 \mathrm{~g}$, including $1180.1 \mathrm{~g}$ plastic material and $647.3 \mathrm{~g}$ metal material.

(4) Applying Eq. (44)-(46), total discarded materials are $674.6 \mathrm{~g}$, including $491.9 \mathrm{~g}$ plastic material and $182.7 \mathrm{~g}$ metal material.

\section{Conclusion}

This research applies the GERT technique to develop a forecasting model to predict the quantity, probability and time of product returns, parts and components 
remanufacturing, material extracting and final disposal, respectively. The steps of using this model involve process mapping, developing GERT stochastic networks and deriving the transfer function of each transfer process and a system equivalence transfer function. The model can be used in any type of product and the remanufacturing process in general. Compared to existing research, the uniqueness of this model is the ability to capture the dynamics of reverse logistics system and remanufacturing process with stochastic features, and be able to predict desired outputs. The main contributions of this research are: developing a new approach and procedure that can be easily adopted in any structure of PRR network; and to our knowledge, this possibly is the first model that can predict the quantity, time and probability of return simultaneously. While we appreciate that the model itself may appear to be mathematically challenging to general practitioners, we hope the result of implement of model could provide the practitioners the predicted return statues so they can use them when planning and scheduling remanufacturing more efficiently.

Our model assumes that the data are extracted from historic data. But how the past data are analysed are into consideration in this paper, which could limit the utilisation of the available information. For future research, it would be worth considering developing a model that integrates with other methodologies that can handle different type of historical data, such as grey theory when part of data are incomplete, fuzzy logic when data are imprecise, and machine learning to improve predictive capacity. This would help to improve the accuracy of forecasting. 


\section{Acknowledgement}

The work was supported by the National Natural Science Foundation of China (Grant No. 71272015).

\section{Reference}

Ahmed, A., Kayis, B. and Amornsawadwatana, S. (2007), “A review of techniques for risk management in projects", Benchmarking: An International Journal, Vol. 14 No. 1, pp. 22-36.

Andrew-munot, M., Ibrahim, R.N. and Junaidi, E. (2015), "An Overview of Used-Products Remanufacturing”, Mechanical Engineering Research, Vol. 5 No. 1, pp. 12-23.

Bayındır, Z.P., Erkip, N. and Güllü, R. (2003), “A model to evaluate inventory costs in a remanufacturing environment", International Journal of Production Economics, Vol. 81-82, pp. 597-607.

De Brito, M.P. and Dekker, R. (2004), “A framework for reverse logistics”, Reverse Logistics, Springer, pp. 3-27.

de Brito, M.P. and van der Laan, E.A. (2009), "Inventory control with product returns: The impact of imperfect information", European Journal of Operational Research, Elsevier B.V., Vol. 194 No. 1, pp. 85-101.

Carrasco-Gallego, R. and Ponce-Cueto, E. (2009), "Forecasting the returns in reusable containers' closed-loop supply chains. A case in the LPG industry.", 3rd International Conference on Industrial Engineering and Industrial Management, 
Barcelona-Terrassa, pp. 311-320.

Christer, A.A.H. and Waller, W.M. (2015), "Delay Time Models of Industrial Inspection Maintenance Problems", Journal of Operational Research Society, Vol. 35 No. 5, pp. 401-406.

Decroix, G.A., Zipkin, P.H., Decroix, A. and Zipkin, P.H. (2005), "Inventory for an Assembly Management System Product or Component Returns with", Management Science, Vol. 51 No. 8, pp. 1250-1265.

Fisher, D.L. and Goldstein, W.M. (1983), "Stochastic PERT networks as models of cognition: Derivation of the mean, variance, and distribution of reaction time using Order-of-Processing (OP) diagrams", Journal of Mathematical Psychology, Vol. 27 No. 2, pp. 121-151.

Fleischmann, M., Bloemhof-Ruwaard, J.M., Dekker, R., van der Laan, E., van Nunen, J. a. E.E. and Van Wassenhove, L.N. (1997), "Quantitative models for reverse logistics: A review”, European Journal of Operational Research, Vol. 103 No. 1, pp. 1-17.

Fleischmann, M., Kuik, R. and Dekker, R. (2002), "Controlling inventories with stochastic item returns: A basic model", European Journal of Operational Research, Elsevier, Vol. 138 No. 1, pp. 63-75.

Fu, N., Lao, H.C. and Varakantham, P. (2015), "Robust Execution Strategies for Project Scheduling with Unreliable Resources and Stochastic Durations", Journal of Scheduling, Vol. Forthcomin. 
García-Alvarado, M.S., Paquet, M. and Chaabane, A. (2015), “On inventory control of product recovery systems subject to environmental mechanisms", International Journal of Production Economics, Vol. 165, pp. 132-144.

Glavic, P. and Lukman, R. (2007), "Review of sustainability terms and their definitions", Journal of Cleaner Production, Vol. 15, pp. 1875-1885.

Govindan, K., Soleimani, H. and Kannan, D. (2014), "Reverse logistics and closed-loop supply chain: A comprehensive review to explore the future", European Journal of Operational Research, Vol. 240 No. 3, pp. 603-626.

Guide Jr., V.D.R. and Srivastava, R. (1997), "Buffering from material recovery uncertainty in a recoverable manufacturing environment", Journal of the Operational Research Society, Vol. 48, pp. 519-529.

Guide, V.D.R. (2000), "Production planning and control for remanufacturing: industry practice and research needs", Journal of Operations Management, Vol. 18 No. 4, pp. $467-483$.

Gungor, A. and Gupta, S.M. (2001), "Disassembly sequence plan generation using a branch-and-bound algorithm", International Journal of Production Research, Vol. 39 No. 3, pp. 481-509.

Hanafi, J., Kara, S. and Kaebernick, H. (2008), "Reverse logistics strategies for end-of-life products", The International Journal of Logistics Management, Vol. 19, pp. 367-388.

Ijomah, W.L., McMahon, C.A., Hammond, G.P. and Newman, S.T. (2007), 
"Development of robust design-for-remanufacturing guidelines to further the aims of sustainable development", International Journal of Production Research, Vol. 45 No. 18-19, pp. 4513-4536.

Ke, J.-C. and Wang, K.-H. (1999), "Cost analysis of the M/M/R machine repair problem with balking, reneging, and server breakdowns", Journal of the Operational Research Society, Vol. 50 No. 3, pp. 275-282.

Kelle, P. and Silver, E. (1989), "Forecasting the returns of reusable containers", Journal of Operations Management, Vol. 8 No. 1, pp. 17-35.

Kerra, W. and Ryan, C. (2001), "Eco-efficiency gains from remanufacturing: A case study of photocopier remanufacturing at Fuji Xerox Australia", Journal of Cleaner Production, Vol. 9 No. 1, pp. 75-81.

Kiesmüller, G.P. and van der Laan, E.A. (2001), “An inventory model with dependent product demands and returns", International Journal of Production Economics, Vol. 72 No. 1, pp. 73-87.

Kosugi, T., Hayashi, A., Matsumoto, T., Akimoto, K., Tokimatsu, K., Yoshida, H., Tomoda, T., et al. (2004), "Time to realization: Evaluation of $\mathrm{CO} 2$ capture technology R\&Ds by GERT (Graphical Evaluation and Review Technique) analyses", Energy, Vol. 29 No. 9-10, pp. 1297-1308.

Kumar, S. and Yamaoka, T. (2007), "System dynamics study of the Japanese automotive industry closed loop supply chain", Journal of Manufacturing Technology Management, Vol. 18 No. 2, pp. 115-138. 
Laan, E. Van Der, Salomon, M., Dekker, R. and Wassenhove, L. Van. (1999), "Inventory with Control in Hybrid Systems Remanufacturing”, Management Science, Vol. 45 No. 5, pp. 733-747.

Lund, R.T. (1984), "Remanufacturing”, Technology Review, Vol. 87 No. 18(2), pp. 19-23, 28-29.

Lye, S.W., Lee, S.G. and Khoo, M.K. (2000), "An algorithm for optimising the servicing of products with constrained, multiple defects", International Journal of Production Research, Vol. 38 No. 10, pp. 2185-2200.

Mason, S.J. (1956a), "Feedback Theory -Further Properties Flow Graphs", Proceedings of the IRE, Vol. 44 No. 7, pp. 920-926.

Mason, S.J. (1956b), "Feedback Theory - Further Properties of Signal Flow Graphs", Proceedings of the IRE, Vol. 44 No. 7, pp. 920-926.

De Meyer, A., Loch, C.H. and Pich, M.T. (2002), "Managing project uncertainty: From variation to chaos”, IEEE Engineering Management Review, Vol. 30 No. 3, pp. 91-98.

Pena-Mora, F. and Li, M. (2001), "Dynamic planning and control methodology for design/build fast-track construction projects", Journal of Construction Engineering and Management, Vol. 1, pp. 1-18.

Pishro-Nik, H. (2016), "Moment Generating Functions", available at: https://www.probabilitycourse.com/chapter6/6_1_3_moment_functions.php (accessed 13 April 2016). 
Pritsker, a. A.B. (1966), GERT: Graphical Evaluation and Review Technique, The Rand Corporation, available at: http://www.rand.org/pubs/research_memoranda/RM4973.

Pritsker, A.A.B. and Happ, W.W. (1966), “GERT:Graphical Evaluation and Review Technique, Part I, Fundamental”, Journal of Industrial Engineering, Vol. 17 No. 5, pp. 267-74.

Richter, K. (1996a), "The extended EOQ repair and waste disposal model", International Journal of Production Economics, Vol. 45 No. 1-3, pp. 443-447.

Richter, K. (1996b), “The EOQ repair and waste disposal model with variable setup numbers", European Journal of Operational Research, Vol. 95 No. 2, pp. 313-324.

Taylor, B.R. and Andrushchenko, I. V. (2014), "Interaction of water temperature and shredders on leaf litter breakdown: A comparison of streams in Canada and Norway", Hydrobiologia, Vol. 721 No. 1, pp. 77-88.

Thierry, M.C. (1993), Strategic production and operations management issues in product recovery management, Erasmus Universiteit/Rotterdam School of Management, Faculteit Bedrijfskunde.

Toktay, L.B., Wein, L.M. and Zenios, S. a. (2000), "Inventory Management of Remanufacturable Products", Management Science, Vol. 46 No. 11, pp. $1412-1426$.

Wikipedia. (2015), "Forecasting", Wikipedia, available at: 
https://en.wikipedia.org/wiki/Forecasting (accessed 24 August 2015).

Xie, J.P., Ren, Y. and Zhao, Z. (2007), "Research on stochastic disassembly network", Journal of Management, Vol. 4 No. 2, pp. 174-17.

Zhao, H., Deshpande, V., Ryan, J.K., Zhao, H. and Ryan, J.K. (2015), "Inventory Sharing and Rationing Dealer Networks Decentralized”, Vol. 51 No. 4, pp. $531-547$

Zhou, L., Naim, M.M., Tang, O. and Towill, D.R. (2006), “Dynamic performance of a hybrid inventory system with a Kanban policy in remanufacturing process", Omega, Vol. 34 No. 6, pp. 585-598.

Zhou, L., Xie, J. and Lin, Y. (2010), "Forecasting Returns in Reverse Logistics using GERT Network Theory"', 5th International Conference on Responsive Manufacturing (sponsored by EPSRC), Ningbo, China. 


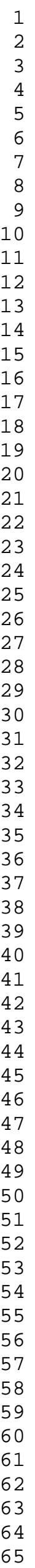

Table 1: Logic nodes for GERT

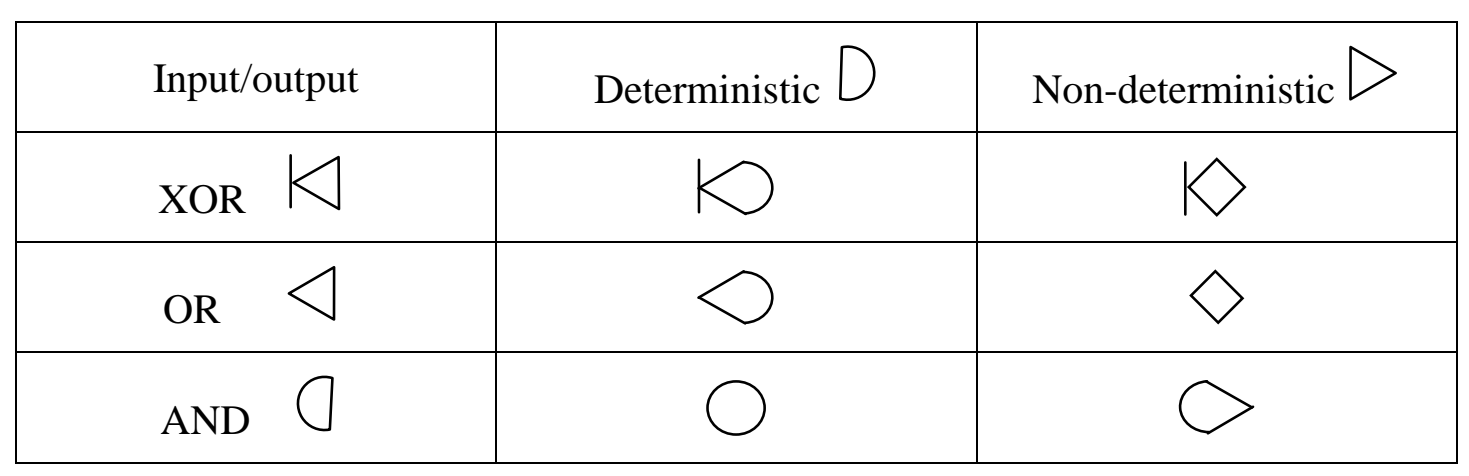


Table 2: Node description

\begin{tabular}{|c|c|}
\hline Node & Indication \\
\hline 0 & Company manufactures the products \\
\hline 1 & First-hand market \\
\hline 2 & Goods returned without using \\
\hline 3 & Product in use in first-hand market \\
\hline 4 & Used product sorting \\
\hline 5 & Second-hand market including refurbishment \\
\hline 6 & Product in use in second-hand market \\
\hline 7 & Classifying products \\
\hline 8 & Product disassembly \\
\hline 9 & Parts refurbishment \\
\hline 10 & Component refurbishment \\
\hline 11 & Recycling for materials \\
\hline 12 & Refurbished parts inventory \\
\hline 13 & Refurbished components inventory \\
\hline 14 & Recycled material inventory \\
\hline 15 & Disposal \\
\hline
\end{tabular}


Table 3: Arrow indication for each process

\begin{tabular}{|c|c|c|}
\hline Arrow & Indication & $\begin{array}{l}\text { Probability } \\
\text { of } \mathrm{P}_{\mathrm{ij}}\end{array}$ \\
\hline $0 \rightarrow 1$ & Product to first-hand market & $100 \%$ \\
\hline $1 \rightarrow 2$ & Product return from the consumer & $1-\mathrm{P}_{1}$ \\
\hline $2 \rightarrow 1$ & Return product to the retailer & $100 \%$ \\
\hline $1 \rightarrow 3$ & Product sold to the customer in use & $\mathrm{P}_{1}$ \\
\hline $3 \rightarrow 3$ & Multiple uses by customer in first-hand market & $\mathrm{P}_{2}$ \\
\hline $3 \rightarrow 4$ & Product enters the sorting point & $\mathrm{P}_{3}$ \\
\hline $4 \rightarrow 5$ & Refurbishment & $\mathrm{P}_{4}$ \\
\hline $4 \rightarrow 7$ & $\begin{array}{l}\text { Product cannot be refurbished and enters product sorting } \\
\text { and testing point }\end{array}$ & $1-\mathrm{P}_{4}$ \\
\hline $5 \rightarrow 6$ & Sold to the customer in second-hand market & $100 \%$ \\
\hline $6 \rightarrow 6$ & Multiple uses by customer in second-hand market & $\mathrm{P}_{5}$ \\
\hline $6 \rightarrow 7$ & Product enters product sorting and testing point & $\mathrm{P}_{6}$ \\
\hline $7 \rightarrow 8$ & Product enters dismantling point & $\mathrm{P}_{7}$ \\
\hline $7 \rightarrow 11$ & Product goes to material extracting process & $\mathrm{P}_{8}$ \\
\hline $7 \rightarrow 15$ & Disposal & $1-\mathrm{P}_{7}-\mathrm{P}_{8}$ \\
\hline $8 \rightarrow 9$ & Enter parts refurbishment process & $\mathrm{P}_{9}$ \\
\hline $8 \rightarrow 10$ & Enter component remanufacturing process & $\mathrm{P}_{10}$ \\
\hline $8 \rightarrow 11$ & Enter material extracting process & $1-\mathrm{P}_{9}-\mathrm{P}_{10}$ \\
\hline
\end{tabular}




\begin{tabular}{|l|l|c|}
\hline $9 \rightarrow 10$ & $\begin{array}{l}\text { Part refurbishment fails so goes to component } \\
\text { remanufacturing process }\end{array}$ & $1-P_{11}^{p}$ \\
\hline $9 \rightarrow 12$ & Enter the parts inventory & $P_{11}^{p}$ \\
\hline $10 \rightarrow 11$ & Refurbishment fails so goes to material extracting & $1-P_{12}^{c}$ \\
\hline $10 \rightarrow 13$ & Enter component inventory & $P_{12}^{c}$ \\
\hline $11 \rightarrow 14$ & Enter material inventory & $P_{13}^{m}$ \\
\hline $11 \rightarrow 15$ & Disposal & $1-P_{13}^{m}$ \\
\hline
\end{tabular}

Where $0 \leq \mathrm{P}_{2}+\mathrm{P}_{3} \leq 1$ and $0 \leq \mathrm{P}_{5}+\mathrm{P}_{6} \leq 1$ 
Table 4: Probabilities and parameters in line with Figure 2

\begin{tabular}{|l|l|l|l|l|}
\hline $\mathrm{P}_{1}=0.95$ & $\mathrm{P}_{2}=0.3$ & $\mathrm{P}_{3}=0.6$ & $\mathrm{P}_{4}=0.3$ & $\mathrm{P}_{5}=0.3$ \\
\hline $\mathrm{P}_{6}=0.6$ & $\mathrm{P}_{7}=0.8$ & $\mathrm{P}_{8}=0.15$ & $\mathrm{P}_{9}=0.7$ & $\mathrm{P}_{10}=0.2$ \\
\hline $\mathrm{t}_{0}=0.6$ & $\mathrm{~N}\left(\mu_{0}=100, \sigma^{2}=30\right)$ & $\mu_{1}=60$ & $\mathrm{t}_{1}=0.1$ & $\mathrm{t}_{2}=0.1$ \\
\hline $\mathrm{t}_{3}=0.2$ & $\mu_{2}=24$ & $\mathrm{t}_{4}=0.1$ & $\mathrm{t}_{5}=0.15$ & $\mathrm{q}_{\mathrm{w}}=1000$ \\
\hline
\end{tabular}


Table 5: Recycling probability and time for parts remanufacturing

\begin{tabular}{|c|c|c|c|c|}
\hline & $\begin{array}{l}\text { Remanufacture to } \\
\text { parts }\left(\mathrm{P}_{11}^{\mathrm{p}}\right)\end{array}$ & $\begin{array}{l}\text { Component } \\
\text { dismantling } \\
1-\mathrm{P}_{11}^{\mathrm{p}}\end{array}$ & $\begin{array}{l}\text { Processing } \\
\text { time } \mathrm{t}_{6}^{\mathrm{p}}\end{array}$ & $\begin{array}{l}\text { Quantity in each } \\
\text { product } \mathrm{q}_{\mathrm{p}}\end{array}$ \\
\hline $\begin{array}{l}\text { Ink cartridge } \\
\text { (IC) }\end{array}$ & 0.7 & 0.3 & 0.4 & 1 \\
\hline $\begin{array}{l}\text { Cleaning } \\
\text { device (CD) }\end{array}$ & 0.3 & 0.7 & 0.5 & 1 \\
\hline Trolley (TO) & 0.5 & 0.5 & 0.4 & 1 \\
\hline $\begin{array}{l}\text { Paper feeder } \\
(\mathrm{PF})\end{array}$ & 0.8 & 0.2 & 0.3 & 1 \\
\hline
\end{tabular}


Table 6: Recycling probability and time expectation for component remanufacturing

\begin{tabular}{|c|c|c|c|c|}
\hline & $\begin{array}{l}\text { Component } \\
\text { remanufacturing } \mathrm{P}_{12}^{\mathrm{c}}\end{array}$ & $\begin{array}{l}\text { Material } \\
\text { recycling } \\
1-\mathrm{P}_{12}^{\mathrm{c}}\end{array}$ & $\begin{array}{l}\text { Processing } \\
\text { time } t_{7}^{c}\end{array}$ & $\begin{array}{l}\text { Quantity in each } \\
\text { part (IC, CD, } \\
\text { TO, PF) } \mathrm{q}_{\mathrm{c}}^{\mathrm{p}}\end{array}$ \\
\hline Screw (SR) & 0.95 & 0.05 & 0.1 & $(4,4,8,4)$ \\
\hline Chip (CI) & 0.8 & 0.2 & 0.8 & $(0,0,1,1)$ \\
\hline $\begin{array}{l}\text { Plastic } \\
\text { component } \\
\text { (PC) }\end{array}$ & 0.6 & 0.4 & 0.3 & $(2,1,3,1)$ \\
\hline $\begin{array}{l}\text { Metal } \\
\text { component } \\
\text { (MC) }\end{array}$ & 0.7 & 0.3 & 0.4 & $(1,2,2,1)$ \\
\hline
\end{tabular}


Table 7: Recycling probability and time for material recycling and disposal

\begin{tabular}{|l|l|l|l|l|}
\hline & Material & Waste & Processing & Weight in each part \\
& renewal $\mathrm{P}_{13}^{\mathrm{m}}$ & $1-\mathrm{P}_{13}^{\mathrm{m}}$ & time $_{8}^{\mathrm{m}}$ & $(\mathrm{SR}, \mathrm{CI}, \mathrm{PC}, \mathrm{MC})$ \\
Plastic & 0.8 & 0.2 & 0.5 & $(0,0.3,0.5,0)$ \\
\hline material & 0.9 & $(\mathrm{~g})$ & $(0.1,0.05,0,0.3)$ \\
\hline Metal & & 0.1 & 0.8 & $\left(\begin{array}{l}\mathrm{m} \\
\text { material }\end{array}\right.$ \\
\hline
\end{tabular}




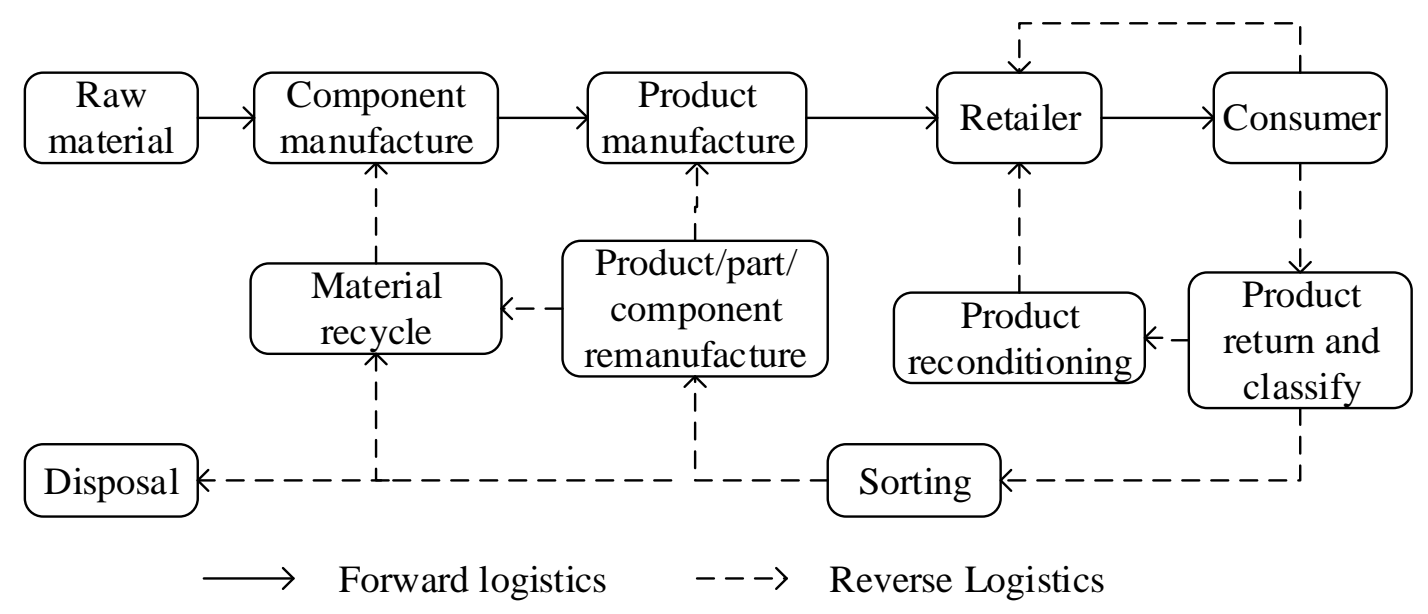

Figure 1: Closed-loop manufacturing and remanufacturing process 


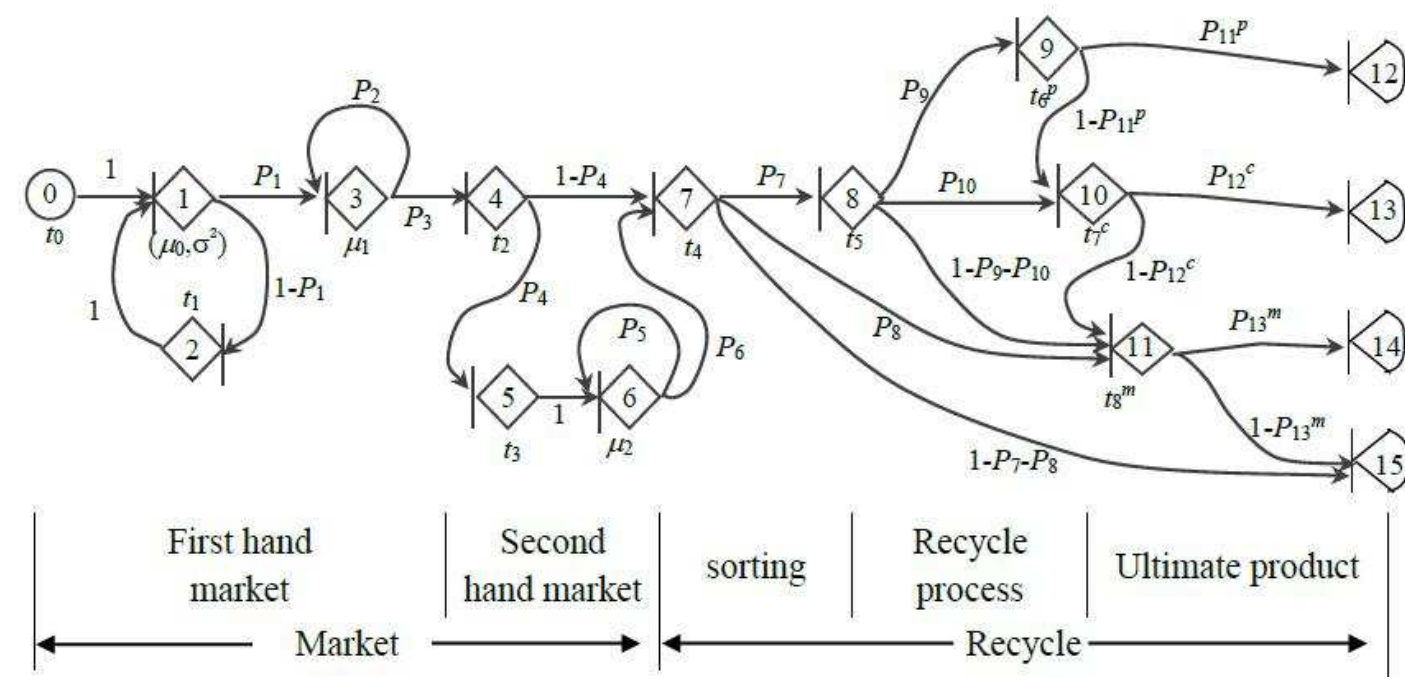

Figure 2: product recycling diagram 


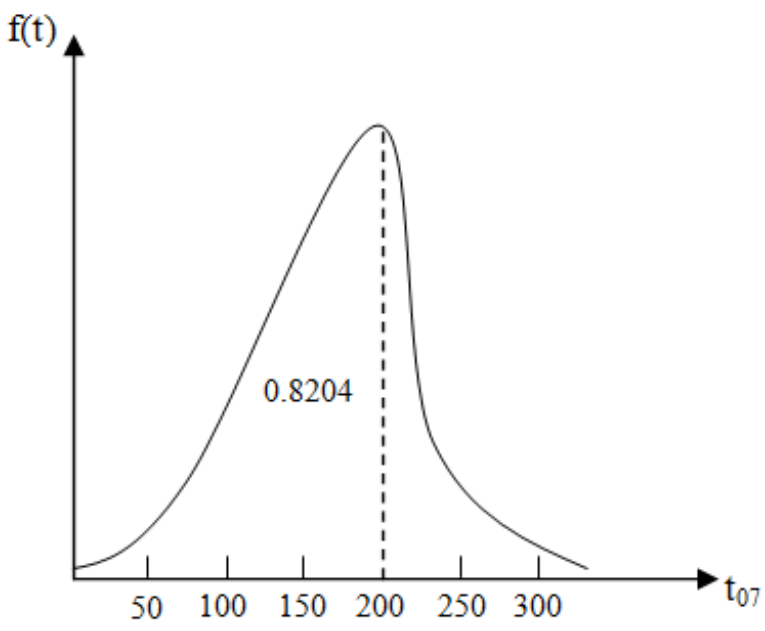

Figure 3. The simulation result of the product return's PDF showing the expected time 200.94 with the probability 0.8204 


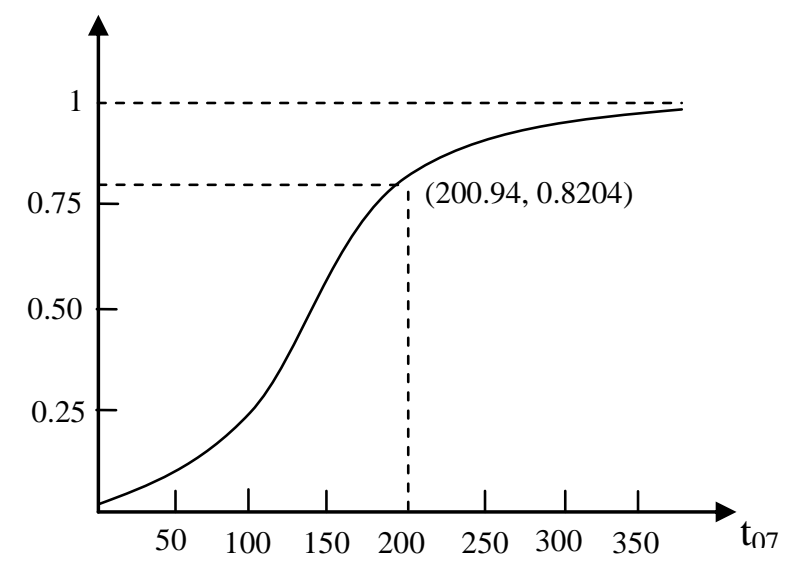

Figure 4. The simulation result of the product return's CPF 